\title{
ON THE SPECTRAL THEORY OF SINGULAR INTEGRAL OPERATORS
}

BY

JOEL DAVID PINCUS(1)

Introduction. The theory of singular integral equations of the form

$$
A(\lambda) x(\lambda)+\frac{\varepsilon}{\pi i} \mathrm{P} \int_{a}^{b} \frac{k(\lambda, \mu)}{\mu-\lambda} x(\mu) d \mu=g(\lambda),
$$

for real $A(\lambda)$ and $k(\lambda, \mu)$, was founded by Hilbert [1], Poincaré [2], and Carleman [3], and in recent years has been pursued energetically by N. I. Muskhelishvili [4] and I. N. Vekua [5] in the Soviet Union.

The operator, acting on $L_{2}(a, b)$, defined by the left-hand side of equation (1), has usually been approached through the study of its so-called dominant part

$$
\left(L_{\varepsilon} x\right)(\lambda)=A(\lambda) x(\lambda)+\frac{\varepsilon}{\pi i} \mathrm{P} \int_{a}^{b} \frac{k(\lambda, \lambda) x(\mu)}{\mu-\lambda} d \mu
$$

which differs from the full operator in (1) by a completely continuous term. However, the classical method of obtaining solutions of equations of the form

$$
\left(L_{\varepsilon}-l\right) x(\lambda)=g(\lambda)
$$

where $l$ is a number, has always been carried out under the restriction

$$
[A(\lambda)-l]^{2}-\varepsilon^{2} k^{2}(\lambda, \lambda) \neq 0, \quad \lambda \in[a, b],
$$

and it can be shown [6], [7] that $l$ is in the spectrum of $L_{\varepsilon}$ precisely if condition (3) is violated.

Nevertheless, despite the fact that the classical work on these equations does not suffice to calculate the generalized eigenfunctions of (2), W. Koppelman and the author were able to guess such eigenfunctions for the special operators

$$
(L x)(\lambda)=\left\{\begin{array}{l}
\lambda x(\lambda)+\frac{\varepsilon}{\pi i} \mathrm{P} \int_{a}^{b} \frac{x(\mu)}{\mu-\lambda} d \mu[8], \\
\frac{1}{\pi i} \mathrm{P}_{1} \int_{a}^{b} \frac{x(\mu)}{\mu-\lambda} d \mu[9],
\end{array}\right.
$$

Received by the editors April 15, 1963.

(1) This work was performed under the auspices of the U.S. Atomic Energy Commission, and is in part based on earlier work done under Contract No. DA-ARO(D)-31-124-6159 at the Courant Institute of Mathematical Sciences. 
and to give a complete spectral representation for them. Both these operators have a simple continuous spectrum, but it proved possible to generalize these results very slightly to certain special operators for which the spectral multiplicity was not greater than two. This was done in independent work by $\mathrm{W}$. Koppelman [10] and the author [11]; both sets of results being based on a common insight generalizing the previous work and using the same identities. However, even the case in which the multiplicity is two was not completely treated. For example, the explicit inversion formulae associated with a spectral representation (in the sense of Mautner) had to be dispensed with in this case [10] because the generalized eigenfunctions were not known.

These singular integral operators were studied initially in connection with the work of K. O. Friedrichs on perturbations of continuous spectra [12],[13] in the hope of providing nontrivial examples in which the perturbation produced a shift of the continuous spectrum and a change in multiplicity; the present work is addressed to the multiplicity problem, and a complete spectral representation for a class of singular integral operators will be given. More precisely, we will investigate under appropriate conditions the spectral representation of the operator, $L_{\varepsilon}$, on $L_{2}(a, b)$ given by

$$
L_{\varepsilon} x(\lambda)=A(\lambda) x(\lambda)+\frac{\varepsilon}{\pi i} \mathbf{P} \int_{a}^{b} \frac{k_{1}(\lambda) k_{2}(\mu)}{\mu-\lambda} x(\mu) d \mu .
$$

That is, we will construct a direct integral Hilbert space $\mathscr{H}^{*}$ which will be characterized in terms of an integer-valued Lebesgue measurable multiplicity function $m(\xi)\left(\xi \in \sigma\left(L_{\varepsilon}\right)\right)$ which we will exhibit explicitly, and a mapping $\mathscr{S}$ (isometric when $L_{\varepsilon}$ is self-adjoint) of $L_{2}(a, b)$ onto $\mathscr{H}^{*}$ given explicitly by a sequence of $m(\xi)$ integral operators whose kernels are generalized eigenfunctions of $L_{\varepsilon}$; furthermore, we will exhibit the transformation inverse to $\mathscr{S}$ explicitly as a sum of integral operators on the components of $\mathscr{H} *$ each of which is given by a kernel formed from the generalized eigenfunctions of $L_{\varepsilon}$.

1. The generalized eigenfunctions. Let

$$
E(l, z, \varepsilon)=\exp \left\{\frac{1}{2 \pi i} \int_{a}^{b} \log \frac{A(\mu)-l-\varepsilon k(\mu)}{A(\mu)-l+\varepsilon k(\mu)} \frac{d \mu}{\mu-z}\right\}\left(^{2}\right)
$$

where $A(\lambda)$ and $k(\lambda) \in C^{1}(a, b)$ and $\operatorname{Im} z \neq 0, \operatorname{Im} l \neq 0$. Let us also introduce the following notation

$$
\begin{aligned}
& E_{ \pm}(l, \lambda, \varepsilon) \equiv E(l, \lambda \pm i 0, \varepsilon), \\
& E^{ \pm}(\xi, z, \varepsilon) \equiv E(\xi \pm i 0, z, \varepsilon),
\end{aligned}
$$

(2) It can be seen that we could have equivalently defined $E(l, z, \varepsilon)$ as a scalar product: $E(l, z, \varepsilon)-1=\left(\sqrt{ }(2 \varepsilon k(\mu)),(\lambda-z)^{-1}\left(L_{\varepsilon}-l\right)^{-1} \sqrt{ }(2 \varepsilon k(\mu))\right)$. 


$$
\begin{aligned}
F(\xi, z, \varepsilon) & \equiv E^{+}(\xi, z, \varepsilon)-E^{-}(\xi, z, \varepsilon), \\
H(l, \lambda, \varepsilon) & \equiv E_{+}(l, \lambda, \varepsilon)-E_{-}(l, \lambda, \varepsilon), \\
F^{ \pm}(\xi, \lambda, \varepsilon) & =\lim _{\eta \downarrow 0} F(\xi, \lambda \pm i \eta, \varepsilon), \\
H_{ \pm}(\xi, \lambda, \varepsilon) \mathbf{1} & =\lim _{\eta \downarrow 0} H(\xi \pm i \eta, \lambda, \varepsilon), \\
E(l, z, \varepsilon) & =\overline{E(l, \bar{z},-\varepsilon)}=\frac{1}{E(l, z,-\varepsilon)}, \\
F(\xi, z,-\varepsilon) & =-\overline{F(\xi, \bar{z}, \varepsilon)}, \\
H(l, \lambda,-\varepsilon) & =-\overline{H(l, \lambda, \varepsilon)},
\end{aligned}
$$

and thus

$$
\overline{F^{+}(\xi, \lambda, \varepsilon)-F^{-}(\xi, \lambda, \varepsilon)}=H_{+}(\xi, \lambda,-\varepsilon)-H_{-}(\xi, \lambda,-\varepsilon) .
$$

The function $E(l, z, \varepsilon)$ has been defined in such a way that

$$
[A(\lambda)-l-\varepsilon k(\lambda)] E_{+}(l, \lambda)-[A(\lambda)-l+\varepsilon k(\lambda)] E_{-}(l, \lambda)=0
$$

for $\lambda \in[a, b]$ and $l$ such that $\operatorname{Im} l \neq 0$. This follows from the Plemelj-Privalov relations [4] which state:

If

$$
\Phi(z)=\frac{1}{2 \pi i} \int_{a}^{b} \frac{x(\mu)}{\mu-z} d \mu,
$$

then

$$
\begin{aligned}
& \Phi(\lambda+i 0)-\Phi(\lambda-i 0)=x(\lambda), \\
& \Phi(\lambda+i 0)+\Phi(\lambda-i 0)=\frac{1}{\pi i} \mathbf{P} \int_{a}^{b} \frac{x(\mu)}{\mu-\lambda} d \mu
\end{aligned}
$$

under smoothness conditions such as $x(\lambda) \in L_{2}(a, b)$.

Now from the Hilbert barrier relation (1.10), we may derive

$$
\begin{aligned}
& {[A(\lambda)-\xi][H(\xi+i \eta, \lambda)-H(\xi-i \eta, \lambda)]} \\
& \quad+\varepsilon k(\lambda)\left[E_{+}(\xi-i \eta, \lambda)+E_{-}(\xi-i \eta, \lambda)-E_{+}(\xi+i \eta, \lambda)-E_{-}(\xi+i \eta, \lambda)\right] \\
& \quad-i \eta[H(\xi+i \eta, \lambda)+H(\xi-i \eta, \lambda)]=0
\end{aligned}
$$

for $\lambda \in(a, b)$ and real $\xi$.

Relation (1.11) follows from (1.10) simply by writing $l=\xi \pm i \eta$, subtracting, and using (1.2) and (1.5).

It can be seen from the following remarks (but will not explicitly be demonstrated here) that the function $H(\xi+i 0, \lambda)-H(\xi-i 0, \lambda)$ is one generalized eigenfunction of the operator 


$$
\left(L_{\varepsilon} x\right)(\lambda)=A(\lambda) x(\lambda)+\frac{\varepsilon}{\pi i} k(\lambda) \mathrm{P} \int_{a}^{b} \frac{x(\mu)}{\mu-\lambda} d \mu
$$

corresponding to the spectral point $\xi$, and it is our purpose now to construct enough other eigenfunctions to furnish a complete spectral representation for $L_{\varepsilon}$ under conditions which will be specified later.

More specifically, we make the guess that there exist real, smooth functions $\left\{\lambda_{j}(\xi)\right\}$ such that $j$ ranges over a finite index set, and

$$
\begin{aligned}
& {[A(\lambda)-\xi] \lim _{\delta \rightarrow 0, \delta>0} \lim _{\eta \rightarrow 0, \eta>0}\left[\frac{H(\xi+i \eta, \lambda)-H(\xi-i \eta, \lambda)}{\lambda+i \delta-\lambda_{j}(\xi)}\right]} \\
& \quad+\frac{\varepsilon}{\pi i} k(\lambda) \lim _{\delta \rightarrow 0, \delta>0} \lim _{\eta \rightarrow 0, \eta>0} \mathrm{P} \int_{a}^{b} \frac{H(\xi+i \eta, \mu)-H(\xi-i \eta, \mu)}{\mu+i \delta-\lambda_{j}(\xi)} \frac{d \mu}{\mu-\lambda}=0
\end{aligned}
$$

and the object of this section will be the determination of such a family of functions $\left({ }^{3}\right)$.

Accordingly, let us divide relation (1.11) through by the unknown function $\lambda+i \delta-\lambda_{j}(\xi), \delta>0$, and observe that, for $\eta>0$ and $\operatorname{Im} z \neq 0$ for $z$ in a neighborhood of an endpoint, $c$, of $(a, b)$ and $l=\xi+i \eta, \eta>0$ it can be shown that [4]

$$
E(l, z, \varepsilon)=O\left(\frac{1}{(z-c)^{r}}\right), \quad r<\frac{1}{2},
$$

and then Cauchy's theorem can be used to conclude that

$$
\text { (1.15) }-\frac{1}{2 \pi i} \int_{a}^{b} \frac{H(\xi+i \eta, \mu)}{\mu i \delta-\lambda_{j}(\xi)} \frac{d \mu}{\mu-z}=\frac{1}{2 \pi i} \oint_{c} \frac{E(\xi+i \eta, \omega)}{\omega+i \delta-\lambda_{j}(\xi)} \frac{d \omega}{\omega-z}
$$

where $C$ is a smooth contour of the type shown.

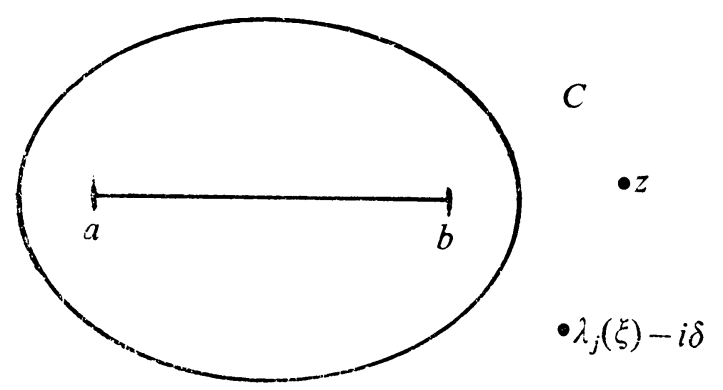

FIGURE 1

But this last integra may be evaluated by the Cauchy residue theorem to give (1.16) $-\frac{1}{2 \pi i} \oint_{c} \frac{E(\xi+i \eta, \omega)}{\omega+i \delta-\lambda_{j}(\xi)} \frac{d \omega}{\omega-z}=\frac{E(\xi+i \eta, z)}{z+i \delta-\lambda_{j}(\xi)}+\frac{E\left(\xi+i \eta, \lambda_{j}(\xi)-i \delta\right)}{\lambda_{j}(\xi)-i \delta-z}$.

(3) These functions, as will be seen, are weak solutions of the eigenvalue integral equation. 
Therefore we may use the Plemelj-Privalov relations to conclude that

$$
\begin{gathered}
-\frac{E_{+}(\xi+i \eta, \lambda)+E_{-}(\xi+i \eta, \lambda)}{\lambda+i \delta-\lambda_{j}(\xi)}-2 \frac{E\left(\xi+i \eta, \lambda_{j}(\xi)-i \delta\right)}{\lambda_{j}(\xi)-i \delta-\lambda} \\
=\frac{1}{\pi i} \mathrm{P} \int_{a}^{b} \frac{H(\xi+i \eta, \mu)}{\mu i \delta-\lambda_{j}(\xi)} \frac{d \mu}{\mu-\lambda}
\end{gathered}
$$

and we have

$$
\begin{aligned}
& {[A(\lambda)-\xi]\left[\frac{H(\xi+i \eta, \lambda)-H(\xi-i \eta, \lambda)}{\lambda+i \delta-\lambda_{j}(\xi)}\right]} \\
& +\frac{\varepsilon}{\pi i} k(\lambda) \mathrm{P} \int_{a}^{b} \frac{H(\xi+i \eta, \mu)-H(\xi-i \eta, \mu)}{\mu+i \delta-\lambda_{j}(\xi)} \frac{d \mu}{\mu-\lambda} \\
& -i \eta\left[\frac{H(\xi+i \eta, \lambda)+H(\xi-i \eta, \lambda)}{\lambda+i \delta-\lambda_{j}(\xi)}\right]-\frac{2 \varepsilon k(\lambda)}{\lambda_{j}(\xi)-i \delta-\lambda} \\
& \cdot\left[E\left(\xi+i \eta, \lambda_{j}(\xi)-i \delta\right)-E\left(\xi-i \eta, \lambda_{j}(\xi)-i \delta\right)\right]=0 .
\end{aligned}
$$

Passing to the limit, we get for $\lambda \neq \lambda_{j}(\xi)$

$$
\begin{aligned}
& {[A(\lambda)-\xi] \lim _{\delta \rightarrow 0} \lim _{\eta \rightarrow 0}\left[\frac{H(\xi+i \eta, \lambda)-H(\xi-i \eta, \lambda}{\lambda+i \delta-\lambda_{j}(\xi)}\right]} \\
& \quad+\frac{\varepsilon}{\pi i} k(\lambda) \lim _{\delta \rightarrow 0} \lim _{\eta \rightarrow 0} \mathrm{P} \int_{a}^{b} \frac{H(\xi+i \eta, \mu)-H(\xi-i \eta, \mu)}{\mu+i \delta-\lambda_{j}(\xi)} \frac{d \mu}{\mu-\lambda} \\
& \quad+\frac{2 \varepsilon k(\lambda)}{\lambda-\lambda_{j}(\xi)} F\left(\xi, \lambda_{j}(\xi)\right)=0 .
\end{aligned}
$$

Thus, subject to the existence of the above limits, we see that a sufficient condition for equation (1.13) to hold is that $\lambda_{j}(\xi)$ be chosen so that $F\left(\xi, \lambda_{j}(\xi)\right)=0$.

With the above remarks as motivation, we pass to a detailed study of the function $F(\xi, z)$.

2. The principal function, $F(\xi, z)$. We assume for the following that $A(\lambda)$ and $k(\lambda) \in C^{1}$, and that $k(\lambda)>0$. (We could equally assume $k(\lambda)<0$.) Furthermore, we assume that the zeros of the functions $R^{\prime \pm}(\lambda)=A^{\prime}(\lambda) \pm \varepsilon k^{\prime}(\lambda)$ partition $[a, b]$ into a finite number of subintervals $\left[\alpha_{i}, \alpha_{i+1}\right]$ where $a=\alpha_{1}<\alpha_{2}<\cdots<\alpha_{n}$ $=b$ and on each of these, one of the following four conditions holds:

Case 1. $\left.\quad R^{+}(\lambda) \uparrow, \quad R^{-}(\lambda) \uparrow\right\}$

$\left.\begin{array}{lll}\text { Case 2. } & R^{+}(\lambda) \uparrow, & R^{-}(\lambda) \downarrow \\ \text { Case 3. } & R^{+}(\lambda) \downarrow, & R^{-}(\lambda) \uparrow\end{array}\right\}$ strict monotonicity is indicated.

Case 4. $\quad R^{+}(\lambda) \downarrow, \quad R^{-}(\lambda) \downarrow$ 
Consider now the function

$$
\begin{aligned}
\int_{a}^{b} \log \frac{A(\mu)-l-\varepsilon k(\mu)}{A(\mu)-l+\varepsilon k(\mu)} \frac{d \mu}{\mu-z} & =\sum_{\kappa=1}^{n-1} \int_{\alpha_{\kappa}}^{x_{\kappa}+1} \log \frac{A(\mu)-l-\varepsilon k(\mu)}{A(\mu)-l+\varepsilon k(\mu)} \frac{d \mu}{\mu-z} \\
& \equiv \sum_{\kappa=1}^{n-1} I(z, l)_{\kappa} .
\end{aligned}
$$

LEMMA 1. For each $\kappa=1,2, \cdots, n-1$ there exist two Lipschitz continuous functions $T_{\kappa}(v, \varepsilon)$ and $S_{\kappa}(v, \varepsilon)$ such that

$$
I(z, l)_{\kappa}=\int_{\sigma_{\kappa}} \log \frac{T_{\kappa}(v, \varepsilon)-z}{S_{\kappa}(v, \varepsilon)-z} \frac{d v}{v-l}
$$

where

$$
\sigma_{\kappa}=\left[\min _{\lambda \in\left[\alpha_{\kappa}, \alpha_{\kappa}+1\right]}(A(\lambda)-\varepsilon k(\lambda)), \max _{\lambda \in\left[\alpha_{\kappa}, \alpha_{\kappa}+1\right]}(A(\lambda)+\varepsilon k(\lambda))\right] .
$$

Proof. Let $v=A(\mu)-\varepsilon k(\mu), \mu \in\left[\alpha_{\kappa}, \alpha_{\kappa+1}\right]$; then we may use the implicit function theorem to solve the equation $A(\mu)-\varepsilon k(\mu)-v=0$ for $\mu$ in terms of $v$ to get a function $\mu_{-}(v, \varepsilon)$ which satisfies $v=A\left(\mu_{-}(v, \varepsilon)\right)-\varepsilon k\left(\mu_{-}(v, \varepsilon)\right)$.

In a similar way we get a function $\mu_{+}(v, \varepsilon)$ which satisfies $v=A\left(\mu_{+}(v, \varepsilon)\right)+\varepsilon k\left(\mu_{+}(v, \varepsilon)\right)$.

Then in Case 1, set:

$$
\begin{aligned}
& S_{\kappa}(v, \varepsilon)= \begin{cases}\alpha_{\kappa+1}, & A\left(\alpha_{\kappa+1}\right)-\varepsilon k\left(\alpha_{\kappa+1}\right)<v<A\left(\alpha_{\kappa+1}\right)+\varepsilon k\left(\alpha_{\kappa+1}\right), \\
\mu_{-}(v, \varepsilon), & A\left(\alpha_{\kappa}\right)-\varepsilon k\left(\alpha_{\kappa}\right)<v<A\left(\alpha_{\kappa+1}\right)-\varepsilon k\left(\alpha_{\kappa+1}\right),\end{cases} \\
& T_{\kappa}(v, \varepsilon)= \begin{cases}\alpha_{\kappa}, & A\left(\alpha_{\kappa}\right)-\varepsilon k\left(\alpha_{\kappa}\right)<v<A\left(\alpha_{\kappa}\right)+\varepsilon k\left(\alpha_{\kappa}\right), \\
\mu_{+}(v, \varepsilon), & A\left(\alpha_{\kappa}\right)+\varepsilon k\left(\alpha_{\kappa}\right)<v<A\left(\alpha_{\kappa+1}\right)+\varepsilon k\left(\alpha_{\kappa+1}\right) ;\end{cases}
\end{aligned}
$$

and for Case 2, set:

$$
\begin{aligned}
& S_{\kappa}(v, \varepsilon)=\alpha_{\kappa+1}, \\
& T_{\kappa}(v, \varepsilon)= \begin{cases}\mu_{-}(v, \varepsilon), & A\left(\alpha_{\kappa}\right)-\varepsilon k\left(\alpha_{\kappa}\right)>v>A\left(\alpha_{\kappa+1}\right)-\varepsilon k\left(\alpha_{\kappa+1}\right), \\
\alpha_{\kappa}, & A\left(\alpha_{\kappa}\right)+\varepsilon k\left(\alpha_{\kappa}\right)>v>A\left(\alpha_{\kappa}\right)-\varepsilon k\left(\alpha_{\kappa}\right), \\
\mu_{+}(v, \varepsilon), & A\left(\alpha_{\kappa+1}\right)+\varepsilon k\left(\alpha_{\kappa+1}\right)>v>A\left(\alpha_{\kappa}\right)+\varepsilon k\left(\alpha_{\kappa}\right) ;\end{cases}
\end{aligned}
$$

for Case 3, set:

$$
\begin{aligned}
& T_{\kappa}(v, \varepsilon)=\alpha_{\kappa}, \\
& S_{\kappa}(v, \varepsilon)= \begin{cases}\mu_{-}(v, \varepsilon), & A\left(\alpha_{\kappa+1}\right)-\varepsilon k\left(\alpha_{\kappa+1}\right)>v>A\left(\alpha_{\kappa}\right)-\varepsilon k\left(\alpha_{\kappa}\right), \\
\alpha_{\kappa+1}, & A\left(\alpha_{\kappa+1}\right)+\varepsilon k\left(\alpha_{\kappa+1}\right)>v>A\left(\alpha_{\kappa+1}\right)-\varepsilon k\left(\alpha_{\kappa+1}\right), \\
\mu_{+}(v, \varepsilon), & A\left(\alpha_{\kappa}\right)+\varepsilon k\left(\alpha_{\kappa}\right)>v>A\left(\alpha_{\kappa+1}\right)+\varepsilon k\left(\alpha_{\kappa+1}\right) ;\end{cases}
\end{aligned}
$$


and finally, for Case 4, set:

$$
\begin{aligned}
& S_{\kappa}(v, \varepsilon)= \begin{cases}\alpha_{\kappa+1}, & A\left(\alpha_{\kappa+1}\right)-\varepsilon k\left(\alpha_{\kappa+1}\right)<v<A\left(\alpha_{\kappa+1}\right)+\varepsilon k\left(\alpha_{\kappa+1}\right), \\
\mu_{+}(v, \varepsilon), & A\left(\alpha_{\kappa+1}\right)+\varepsilon k\left(\alpha_{\kappa+1}\right)<v<A\left(\alpha_{\kappa}\right)+\varepsilon k\left(\alpha_{\kappa}\right),\end{cases} \\
& T_{\kappa}(v, \varepsilon)= \begin{cases}\alpha_{\kappa}, & A\left(\alpha_{\kappa}\right)-\varepsilon k\left(\alpha_{\kappa}\right)<v<A\left(\alpha_{\kappa}\right)+\varepsilon k\left(\alpha_{\kappa}\right), \\
\mu_{-}(v, \varepsilon), & A\left(\alpha_{\kappa+1}\right)-\varepsilon k\left(\alpha_{\kappa+1}\right)<v<A\left(\alpha_{\kappa}\right)-\varepsilon k\left(\alpha_{\kappa}\right) .\end{cases}
\end{aligned}
$$

The proof of the lemma is now obvious( $\left.{ }^{4}\right)$ and involves only substitution of these functions and integration by parts.

Let us note here for future use that $S_{\kappa}\left(\alpha_{\kappa}, \varepsilon\right)=T_{\kappa}\left(\alpha_{\kappa}, \varepsilon\right)$ and $S_{\kappa}\left(\alpha_{\kappa+1}, \varepsilon\right)=T_{\kappa}\left(\alpha_{\kappa+1}, \varepsilon\right)$, and if the functions $R^{ \pm}(\lambda)$ satisfy the conditions of the correct combination of cases on the intervals $\left(\alpha_{k}, \alpha_{k+1}\right)$ and $\left(\alpha_{k+1}, \alpha_{k+2}\right)$ then it is possible to find intervals of

$$
\xi \in \sigma \equiv \bigcup_{\kappa=1}^{n-1} \sigma_{\kappa}=\left[\min _{\mu \in(a, b)} R^{-}(\mu), \max _{\mu \in(a, b)} R^{+}(\mu)\right]
$$

for which $S_{\kappa}(\xi, \varepsilon)=T_{\kappa+1}(\xi, \varepsilon)$. For all other $\xi \in \sigma$, we will have

$$
T_{1}(\xi)<S_{1}(\xi)<T_{2}(\xi)<\cdots<T_{n-1}(\xi)<S_{n-1}(\xi) .
$$

If $S_{\kappa}(\xi, \varepsilon)$ and $T_{\kappa}(\xi, \varepsilon)$ are extended to $\sigma$ by setting them equal outside $\sigma_{\kappa}$ to the constant values they assume at the endpoints of $\sigma_{\kappa}$, then

$$
E(l, z, \varepsilon)=\exp \left\{\frac{1}{2 \pi i} \int_{\sigma} \log \prod_{\kappa=1}^{n-1} \frac{T_{\kappa}(v, \varepsilon)-z}{S_{\kappa}(v, \varepsilon)-z} \frac{d v}{v-l}\right\}
$$

and we may define the "multiplicity polynomials"

$$
\begin{aligned}
& T(v, z)=\prod_{\kappa=1}^{n-1}\left[T_{\kappa}(v, \varepsilon)-z\right], \\
& S(v, z)=\prod_{\kappa=1}^{n-1}\left[S_{\kappa}(v, \varepsilon)-z\right] .
\end{aligned}
$$

Now it is easy to show that

$$
\begin{aligned}
& E_{+}(\xi, z, \varepsilon)_{\kappa}=\exp \left\{\frac{1}{2 \pi i} \int_{\alpha_{\kappa}}^{\alpha_{\kappa+1}} \log \left|\frac{A(\mu)-\xi-\varepsilon k(\mu)}{A(\mu)-\xi+\varepsilon k(\mu)}\right| \frac{d \mu}{\mu-z}\right\} \sqrt{\left(\frac{T_{\kappa}(\xi, \varepsilon)-z}{S_{\kappa}(\xi, \varepsilon)-z}\right),} \\
& E_{-}(\xi, z, \varepsilon)_{\kappa}=\exp \left\{\frac{1}{2 \pi i} \int_{\alpha_{\kappa}}^{\alpha_{\kappa}+1} \log \left|\frac{A(\mu)-\xi-\varepsilon k(\mu)}{A(\mu)-\xi+\varepsilon k(\mu)}\right| \frac{d \mu}{\mu-z}\right\} \sqrt{\left(\frac{S_{\kappa}(\xi, \varepsilon)-z}{T_{\kappa}(\xi, \varepsilon)-z}\right)}
\end{aligned}
$$

by evaluating $\arg (A(\mu)-\xi \pm i \eta \pm \varepsilon k(\mu))$ as $\eta \rightarrow 0$.

(4) We might also have proceeded directly from the relation

$$
\log \{(A(\mu)-l-\varepsilon k(\mu)) /(A(\mu)-l+\varepsilon k(\mu)\}
$$

$$
=\pi^{-1} \int_{\sigma} \arg \{(A(\mu)-v-i 0-\varepsilon k(\mu)) /(A(\mu)-v-i 0-\varepsilon k(\mu))\}(d v /(\nu-l)) \text {. }
$$


Therefore, we may deduce that

$$
F(\xi, z)=\frac{T(\xi, z)-S(\xi, z)}{\sqrt{ }(S(\xi, z) T(\xi, z))} \exp \left\{\frac{1}{2 \pi i} \int_{a}^{b} \log \left|\frac{A(\mu)-\xi-\varepsilon k(\mu)}{A(\mu)-\xi+\varepsilon k(\mu)}\right| \frac{d \mu}{\mu-z}\right\}
$$

and it is now clear that the roots of $F(\xi, z)$ are the roots of the function

$$
W(\xi, z)=\frac{T(\xi, z)-S(\xi, z)}{\sqrt{ }(S(\xi, z) T(\xi, z))} .
$$

Now if we call $\theta(\xi)$ the set of indices $\kappa$ for which $S_{\kappa}(\xi, z)=T_{\kappa}(\xi, z)$ or $S_{\kappa}(\xi, z)=T_{\kappa+1}(\xi, z)$, then

$$
W(\xi, z)=\frac{\prod_{j \notin \theta(\xi)}\left(T_{j}(\xi)-z\right)-\prod_{j \notin \theta(\xi)}\left(S_{j}(\xi)-z\right)}{\left.\sqrt{(} \prod_{j \notin \theta(\xi)}\left(S_{j}(\xi)-z\right)\left(T_{j}(\xi)-z\right)\right)} .
$$

Thus the number of zeros of $W(\xi, z)$ depends upon $\xi$. We will call this integer $m(\xi)-1$ and observe that $0<m(\xi)<n$ and the roots $\lambda_{1}(\xi), \cdots, \lambda_{m(\xi)-1}(\xi)$ are easily seen to be both real and simple for each fixed $\xi$.

It is possible to give a formula for $m(\xi)$ that does not involve $S(\xi, z)$ or $T(\xi, z)$.

Let us simply note that

$$
\sum_{\kappa=1}^{n-1} \log \frac{T_{\kappa}(\xi)-z}{S_{\kappa}(\xi)-z}=\frac{1}{\pi} \int_{a}^{b} \arg \frac{A(\mu)-\xi-i 0-\varepsilon k(\mu)}{A(\mu)-\xi-i 0+\varepsilon k(\mu)} \frac{d \mu}{\mu-z}
$$

and for fixed $\xi$ the contributions to the integral come from a set of intervals on each of which

$$
\frac{A(\mu)-\xi-\varepsilon k(\mu)}{A(\mu)-\xi+\varepsilon k(\mu)}<0 .
$$

The number of nonvanishing terms on the left of (2.11) will simply be equal to the number of such intervals, and this number will be equal to $m(\xi)$. It follows from this representation that $m(\xi)$ is Lebesgue measurable.

Lemma 2. If $f(\lambda) \in C^{\infty}(a, b)$ and we define

$$
T f=\lim _{\eta \rightarrow 0, \eta>0} \int_{a}^{b}[H(\xi+i \eta, \lambda,-\varepsilon)-H(\xi-i \eta, \lambda,-\varepsilon)] f(\lambda) d \lambda,
$$

then

$$
T f=\lim _{\eta \rightarrow 0, \eta>0}{ }_{a}^{b}[F(\xi, \lambda+i \eta)-F(\xi, \lambda-i \eta)] f(\lambda) d \lambda .
$$

Proof. This theorem concerns an interchange of orders in a limiting process (if it is rewritten in terms of the $E(l, z)$-functions). The assertion makes sense formally because of the identity 


$$
H_{+}(\xi, \lambda,-\varepsilon)-H_{-}(\xi, \lambda,-\varepsilon)=F^{+}(\xi, \lambda, \varepsilon)-F^{-}(\xi, \lambda, \varepsilon) ;
$$

nevertheless, the result is not immediate because the required uniform estimates for justification of a limit interchange do not seem to follow easily. We will, accordingly, pursue a less direct route in the proof, and will arrive at the result finally by considering the question in a somewhat widened context, and invoking the following theorem of H. G. Tillman [14].

Definition. A distribution $T$ on $R$ with values in a locally convex vector space $F$ is a continuous linear mapping of the space $\mathscr{D}=C_{0}^{\infty}(R)$ into the space $F$.

TheOREM (TILLMAN). There exists a natural isomorphism of the space of $F$-valued distributions $\hat{T}$ with compact support and the space of F-valued holomorphic functions $t(\cdot)$ in the complement of a compact set $K \subset R$ with the property that $t$ is also holomorphic at infinity and satisfies a condition $p(t(z)) \leqq M_{p}\left\{d(z, K)^{-m_{p}}+d(z, K)^{-1}\right\}$ for any continuous seminorm $p$ in $F$. $(d(z, K)$ is the distance between $z$ and $K$.) This isomorphism is defined by the formulas

$$
\begin{aligned}
& t(z)=\frac{1}{2 \pi i} \hat{T}_{s}\left[(s-z)^{-1}\right], \\
& \hat{T} f=\lim _{\mathscr{R} \rightarrow 0} \int f(s)[t(s+i \varepsilon)-t(s-i \varepsilon)] d s, \quad f \in \mathscr{D} .
\end{aligned}
$$

To begin the proof, we note

Observation 1. T, defined by (2.11a), is a distribution with values in $L_{2}(\sigma)$. Clearly, in order to substantiate this observation it will suffice to demonstrate that $T$ is a bounded map of $L_{2}(a, b)$ into $L_{2}(\sigma)$; and we proceed to a demonstration of this fact $\left(^{5}\right)$.

Let us define

$$
U f=-\frac{1}{2 \pi i} \lim _{\eta \rightarrow 0, \eta>0} \int_{a}^{b} \frac{\bar{H}(\xi+i \eta, \lambda)-\bar{H}(\xi-i \eta, \lambda)}{\sqrt{ }(k(\lambda)) B(\xi)} f(\lambda) d \lambda
$$

where

$$
\left.B(\xi)=\sqrt{(2 \varepsilon} \sum_{j=1}^{n-1} S_{j}(\xi, \varepsilon)-T_{j}(\xi, \varepsilon)\right) .
$$

Then $U$ generates an isometry from a certain linear manifold, $D(U)\left(^{6}\right)$, which is generated by the elements $\{\sqrt{ } 2 \varepsilon k(\lambda), H(\omega, \lambda) / \sqrt{ } 2 \varepsilon k(\lambda)\}$ as $\omega$ ranges over the complement of $\sigma$, to the manifold generated by the image of these functions; namely (as we can easily see), the manifold generated by

(5) This demonstration is related to a somewhat different construction in (10) which does not quite suffice to prove this observation.

(6) $D(u)$ is the smallest closed invariant subspace which contains $\sqrt{ }(2 \varepsilon k(\lambda))$, because $\left(\left(L_{\varepsilon}-\omega\right)^{-1} \sqrt{ }(2 \varepsilon k(\lambda))=H(\omega, \lambda) / \sqrt{ }(2 \varepsilon k(\lambda))\right.$. 


$$
\left\{\frac{B(\xi)}{\sqrt{ }(2 \varepsilon)}, \frac{B(\xi)}{\sqrt{ }(2 \varepsilon)} \cdot \frac{1}{\xi-\omega}\right\}
$$

which we call $D\left(U^{-1}\right)$.

The assertion that $U$ restricted to $D(U)$ is an isometry means that certain scalar products are equal, and these equalities follow easily. For example, we can show that

$$
\int_{a}^{b} \frac{H(\omega, \lambda)}{\sqrt{ }(2 \varepsilon k(\lambda))} \cdot \sqrt{ }(2 \varepsilon k(\lambda)) d \lambda=\int_{\sigma} \frac{B(\xi)}{\sqrt{ }(2 \varepsilon)} \frac{1}{\xi-\omega} \cdot \frac{B(\xi)}{\sqrt{ }(2 \varepsilon)} d \xi
$$

Now we shall construct an extension of $U^{-1}$ which shall be such that the closure of its range will be all of $L_{2}(a, b)$. For this purpose, consider the vectorvalued functions

$$
\boldsymbol{g}(\xi)=\left(g_{0}(\xi), g_{1}(\xi), \cdots, g_{m(\xi)-1}(\xi)\right)
$$

where each component $g_{\kappa}(\xi)$ is complex valued and the number of components on the right-hand side is $m(\xi)$. Each $g_{\kappa}(\xi)$ is defined on the measurable set $\tau_{\kappa}=\{\xi \mid m(\xi) \geqq \kappa\}$, and we let $\mathscr{H}^{*}$ be the set of all $g(\xi)$ with measurable components for which

$$
(g, g) \equiv \int_{\sigma} \sum_{0}^{m(\xi)-1}\left|g_{\kappa}(\xi)\right|^{2} d \xi<\infty
$$

A scalar product is introduced in $\mathscr{H}^{*}$ by setting

$$
(f, g)=\int_{\sigma}^{m(\xi)-1} \sum_{0}(\xi) \overline{g_{k}(\xi)} d \xi
$$

and the equivalence classes in $\mathscr{H}^{*}$ with respect to functions of vanishing norm form a Hilbert space which we again call $\mathscr{H}^{*}$.

If we let, for $\xi \in \tau_{j}$,

$$
\mu_{j}(\xi)=\frac{T\left(\xi, \lambda_{j}(\xi)\right)}{S^{\prime}\left(\xi, \lambda_{j}(\xi)\right)-T^{\prime}\left(\xi, \lambda_{j}(\xi)\right)}, \quad j=1, \cdots, m(\xi),
$$

we can easily show that $\mu_{j}(\xi) \geqq 0$ follows from $T_{1}(\xi, \varepsilon)<S_{1}(\xi, \varepsilon)<\cdots<S_{n-1}(\xi)$ $<T_{n-1}(\xi)$; and we may define

$$
\begin{aligned}
& U_{\text {extended }}^{-1}(g)=\frac{1}{2 \pi i} \lim _{\eta \rightarrow 0, \eta>0}\left(\int_{\sigma} \frac{F(\xi, \lambda+i \eta)-F(\xi, \lambda-i \eta)}{B(\xi) \sqrt{ } k(\lambda)} g_{0}(\xi) d \xi\right.
\end{aligned}
$$

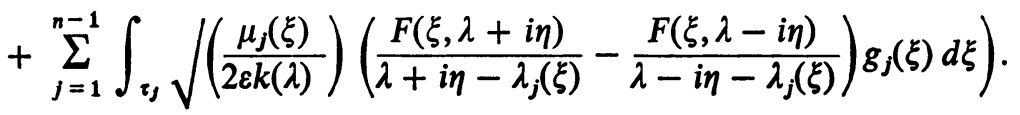


Then, if

$$
\begin{aligned}
\boldsymbol{g}_{z} & \equiv\left(\frac{F(\xi, \bar{z})}{B(\xi) / 2 \varepsilon}, \frac{F(\xi, \bar{z})}{z-\lambda_{1}(\xi)} \sqrt{ } \mu_{1}(\xi), \cdots, \frac{F(\xi, \bar{z})}{z-\lambda_{m(\xi)-1}(\xi)} \sqrt{ } \mu_{m(\xi)-1}(\xi)\right) \\
U_{\text {extended }}^{-1}\left(g_{z}\right) & =\frac{1}{2 \pi i} \lim _{\eta \rightarrow 0, \eta>0}\left(\int_{\sigma} \frac{F(\xi, \lambda+i \eta)-F(\xi, \lambda-i \eta)}{B(\xi) \sqrt{ } k(\lambda)} \frac{F(\xi, \bar{z})}{B(\xi) / 2 \varepsilon} d \xi\right. \\
& \left.+\sum_{j=1}^{n-1} \int_{\sigma} \frac{\mu_{j}(\xi)}{\sqrt{k(\lambda)}}\left(\frac{F(\xi, \lambda+i \eta)}{\lambda+i \eta-\lambda_{j}(\xi)}-\frac{F(\xi, \lambda-i \eta)}{\lambda-i \eta-\lambda_{j}(\xi)}\right) \frac{F(\xi, \bar{z})}{z-\lambda_{j}(\xi)} d \xi\right) .
\end{aligned}
$$

In order to evaluate this function explicitly, it is only necessary to notice the following elementary fact:

$$
\frac{T(v, y)}{T(v, y)-S(v, y)}-\frac{T(v, x)}{T(v, x)-S(v, x)}=\frac{T(v, x) S(v, y)-T(v, y) S(v, x)}{[T(v, y)-S(v, y)][T(v, x)-S(v, x)]}
$$

and to expand each term of the left-hand side in partial fractions. We obtain

$$
\begin{aligned}
\frac{T(v, x)}{S(v, x)-T(v, x)}= & \sum_{j=1}^{m(v)-1} \frac{T\left(v, \lambda_{j}(v)\right)}{S^{\prime}\left(v, \lambda_{j}(v)\right)-T^{\prime}\left(v, \lambda_{j}(v)\right)} \cdot \frac{1}{x-\lambda_{j}(v)} \\
& +\frac{x}{\sum_{j=1}^{m(v)-1}\left[S_{j}(v)-T_{j}(v)\right]}+\text { a term constant in } x .
\end{aligned}
$$

Therefore

$$
\frac{1}{x-y} \frac{T(v, x) S(v, y)-T(v, y) S(v, x)}{[T(v, x)-S(v, x)][T(v, y)-S(v, y)]}=\sum_{j=1}^{m(v)-1} \frac{\sqrt{ } \mu_{j}(v)}{y-\lambda_{j}(v)} \cdot \frac{\sqrt{ } \mu_{j}(v)}{x-\lambda_{j}(v)}
$$

$$
+\frac{1}{\sum_{j=1}^{m(v)-1}\left[T_{j}(v, \varepsilon)-S_{j}(v, \varepsilon)\right]}
$$

But by Lemma 1 (2.4),

$$
E(l, z)=\exp \left\{\frac{1}{2 \pi i} \int_{\sigma} \log \frac{T(v, z)}{S(v, z)} \frac{d v}{v-l}\right\},
$$

so that the Plemelj-Privalov relations imply

$$
T(v, z) E^{+}(v, z)-S(v, z) E^{-}(v, z)=0
$$

which, in turn, implies, after a little algebra,

$$
\begin{aligned}
\frac{T\left(v, z_{1}\right) S\left(v, z_{2}\right)-T\left(v, z_{2}\right) S\left(v, z_{1}\right)}{\left[S\left(v, z_{1}\right)-T\left(v, z_{1}\right)\right]\left[S\left(v, z_{2}\right)-T\left(v, z_{2}\right)\right]} F\left(v, z_{1}\right) \overline{F\left(v, \bar{z}_{2}\right)} \\
=E^{+}\left(v, z_{1}\right) \overline{E^{-}\left(v, \bar{z}_{2}\right)}-E^{-}\left(v, z_{1}\right) \overline{E^{+}\left(v, \bar{z}_{2}\right)} .
\end{aligned}
$$


But now we can use the Cauchy residue theorem to get

$$
\begin{aligned}
& \frac{1}{2 \pi i} \int_{\sigma}\left\{E\left(v+i 0, z_{1}\right) \bar{E}\left(v-i 0, \bar{z}_{2}\right)-E\left(v-i 0, z_{1}\right) \bar{E}\left(v+i 0, \bar{z}_{2}\right)\right\} d v \\
& \quad=\frac{1}{2 \pi i} \oint_{C} E\left(l, z_{1}\right) \overline{E\left(l, \bar{z}_{2}\right)} d l=\frac{1}{\pi i} \int_{a}^{b} \varepsilon k(\mu)\left\{\frac{1}{\mu-z_{2}}-\frac{1}{\mu-z_{1}}\right\} d \mu
\end{aligned}
$$

from which we obtain, finally

$$
U_{\text {extended }}^{-1}\left(g_{z}\right)=\frac{\sqrt{ }(2 \varepsilon k(\lambda))}{\lambda-z}
$$

Hence, if we consider $U_{\text {extended }}^{-1}$ as being defined on the manifold generated by

$$
\left\{D\left(U^{-1}\right), g_{z}^{(1)}, \cdots, g_{z}^{m(\xi)-1}\right\}=D\left(U_{\text {extended }}^{-1}\right)
$$

we see, since the functions $\{\sqrt{ }(2 \varepsilon k(\lambda)) /(\lambda-z)\}$ are dense in $L_{2}(a, b)$, that the closure of its range is $L_{2}(a, b)$.

Moreover, we can show that $U_{\text {extended }}^{-1}$ restricted to $D\left(U_{\text {extended }}^{-1}\right)$ is an isometry since all the scalar products involved in this assertion can be calculated by using the foregoing identities and the residue theorem. The required calculation is tedious but elementary.

Accordingly, $U_{\text {extended }}^{-1}$ has an inverse, which we will call $U_{\text {extended, }}$ which can be continuously defined on all of $L_{2}(a, b)$. However, since

$$
U\left\{\frac{\sqrt{ }(2 \varepsilon k(\lambda))}{\lambda-z}\right\}=\frac{F(\xi, \bar{z})}{B(\xi) / 2 \varepsilon}
$$

we must have $P U_{\text {extended }}=U$, where $P$ is the projection from $\mathscr{H}^{*}$ onto its first component.

Thus, $U$ is a bounded map from $L_{2}(a, b)$ into $L_{2}(\sigma)$; and it then follows that $T$ is bounded. Hence, a direct use of Tillman's theorem now proves the lemma.

REMARK. A real convex lower-semicontinuous functional $p(h)$ on a Hilbert space $H$ is bounded by the Hilbert norm, i.e. there exists a constant $M_{p}$ such that $p(h) \leqq M_{p}\|h\|$ for all $h \in H$.

Thus all semi-norms on $H$ [15] are bounded by the Hilbert norm.

But the relations

$$
-\frac{1}{2 \pi i} \int_{a}^{b} \frac{H(\xi+i \eta, \mu)}{\mu-z} d \mu=\frac{1}{2 \pi i} \oint_{c} \frac{E(\xi+i \eta, \omega)}{\omega-z} d \omega=E(\xi+i \eta, z)-1
$$

imply that

$$
U \cdot \sqrt{ }(k(s))\left[(s-z)^{-1}\right]=\frac{F(\xi, \bar{z})}{B(\xi) / \sqrt{ }(2 \varepsilon)}
$$


But by (2.13) and (2.14), we can assert

$$
\int_{\sigma} \frac{|F(\xi, z)|^{2}}{B^{2}(\xi) / 2 \varepsilon} d \xi \leqq \int_{\sigma} \frac{|F(\xi, z)|^{2}}{z-\bar{z}} \frac{T(\xi, z) S(\xi, \bar{z})-T(\xi, \bar{z}) S(\xi, z)}{[T(\xi, z)-S(\xi, z)][T(\xi, \bar{z})-S(\xi, \bar{z})]} d \xi
$$

and by (2.15)

$$
\int_{a} \frac{|F(\xi, z)|^{2}}{B^{2}(\xi) / 2 \varepsilon} d \xi \leqq 2 \int_{a}^{b} \varepsilon k(\mu) \frac{1}{|\mu-z|^{2}} d \mu \leqq C d(z,[a, b])^{-2} .
$$

Thus we have another proof of the lemma from Tillman's theorem and a direct estimate of the norm of the indicatrix function.

We may also conclude that, for $\delta>0$,

$$
\begin{aligned}
\lim _{\eta \rightarrow 0, \eta>0} & \int_{a}^{b} \frac{\overline{H(\xi+i \eta, \lambda)}-\overline{H(\xi-i \eta, \lambda)}}{\lambda+i \delta-\lambda_{j}(\xi)} f(\lambda) d \lambda \\
& =\lim _{\eta \rightarrow 0, \eta>0} \int_{a}^{b} \frac{\overline{F(\xi, \lambda+i \eta)}-\overline{F(\xi, \lambda-i \eta)}}{\lambda+i \delta-\lambda_{j}(\xi)} f(\lambda) d \lambda \text { a.a. } \xi \in \sigma .
\end{aligned}
$$

Now we turn to

LEMMA $3\left({ }^{7}\right)$. If $g(\xi) \in C^{\infty}(\sigma)$, and we define

$$
\hat{T} g=\lim _{\eta \rightarrow 0, \eta>0} \int_{\sigma}(F(\xi, \lambda+i \eta)-F(\xi, \lambda-i \eta)) g(\xi) d \xi,
$$

then

$$
\hat{T} g=\lim _{\eta \rightarrow 0, \eta>0} \int_{\sigma}(H(\xi+i \eta, \lambda)-H(\xi-i \eta, \lambda)) g(\xi) d \xi
$$

This follows in much the same manner as Lemma 2. First we observe that

$$
\mathscr{T} \equiv \frac{1}{\sqrt{ } k(\lambda)} \hat{T} \cdot \frac{1}{B(\xi)}
$$

is a bounded operator on $L_{2}(\sigma)$ because $\mathscr{T}=U^{-1}$ on $\mathscr{D}\left(U^{-1}\right)$ where $U$ is defined in (2.12); this identity follows from an explicit computation of the images obtained when $\mathscr{T}$ acts on the elements generating $\mathscr{D}\left(U^{-1}\right)$. If we further observe that the set of functions $\{B(\xi) g(\xi)\}$ is dense in $L_{2}(\sigma)$ when $g(\xi)$ ranges over $L_{2}(\sigma)$, we can conclude that $\hat{T}$ has a bounded extension defined on all of $L_{2}(\sigma)$.

Now let us again compute the indicatrix function $\hat{T}\left[(s-l)^{-1}\right]$. To do this, simply observe that

$$
\frac{1}{2 \pi i} \int_{\sigma} \frac{F(\xi, \mu+i \eta)}{\xi-l} d \xi=\frac{1}{2 \pi i} \oint_{c} \frac{E(\omega, \mu+i \eta)}{\omega-l} d \omega=E(l, \mu+i \eta)-1
$$

(7) Lemma 3 will not be used in the proof of the representation theorem. 
where $C$ is a smooth contour enclosing $\sigma$, and therefore

$$
\hat{T}\left[(s-l)^{-1}\right]=H(l, s) .
$$

Now, we again invoke Tillman's theorem and the lemma is proved.

REMARK. We could have given another proof of the lemma from the identity (2.16) $\left(l_{2}-l_{1}\right) H\left(l_{1}, \lambda\right) \bar{H}\left(l_{2}, \lambda\right)=2 \varepsilon k(\lambda)\left\{E_{+}\left(I_{1}, \lambda\right) \overline{E_{-}\left(l_{2}, \lambda\right)}-E_{-}\left(l_{1}, \lambda\right) \overline{E_{+}\left(l_{2}, \lambda\right)}\right\}$

which follows immediately from the basic Hilbert relation (1.10), and which in turn enables us to conclude that

$$
\int_{a}^{b} \frac{\overline{H\left(l_{2}, \lambda, \varepsilon\right)} H\left(l_{1}, \lambda, \varepsilon\right)}{2 \varepsilon k(\lambda)} d \lambda=\frac{1}{l_{1}-l_{2}} \int_{\sigma} B(v)\left\{\frac{1}{v-l_{2}}-\frac{1}{v-l_{1}}\right\} d v
$$

from which an estimate of the norm of the indicatrix of $\hat{T}$ follows. Again we may also conclude that, for $\delta>0, g(\xi) \in C^{\infty}(\sigma)$

$$
\begin{aligned}
\lim _{\eta \rightarrow 0, \eta>0} & \int_{\sigma} \frac{F(\xi, \lambda+i \eta)-F(\xi, \lambda-i \eta)}{\lambda+i \delta-\lambda_{j}(\xi)} g(\xi) d \xi \\
& =\lim _{\eta \rightarrow 0, \eta>0} \int_{\sigma} \frac{H(\xi+i \eta, \lambda)-H(\xi-i \eta, \lambda)}{\lambda+i \delta-\lambda_{j}(\xi)} g(\xi) d \xi \text { a.a. } \lambda \in(a, b) .
\end{aligned}
$$

LEMMA 4. Let $G$ denote the set of those functions in $C^{\infty}(\sigma)$ which vanish identically in neighborhoods of the points $A\left(\alpha_{k}\right) \pm \varepsilon k\left(\alpha_{k}\right)$ and $A\left(\alpha_{k+1}\right) \pm \varepsilon k\left(\alpha_{k+1}\right)$, $k=1,2, \cdots, n$.

Then the mappings $M_{j}^{ \pm}$generated by

$$
M_{j}^{ \pm}(g)=\lim _{\delta \downarrow 0} \lim _{\eta \downarrow 0} M_{\eta, \delta}^{j}(g)=\lim _{\delta \downarrow 0} \lim _{\eta \downarrow 0} \int_{\tau_{j}} \frac{F(\xi, \lambda \pm i \eta)}{\lambda+i \delta-\lambda_{j}(\xi)} g(\xi) d \xi
$$

map $G$ into $L_{1}(a, b)$, and

$$
\lim _{\delta \downarrow 0} \lim _{\eta \downarrow a} \int_{a}^{b}\left|M_{\eta, \delta}^{j}(g)-M_{j}(g)\right| d \lambda=0 .
$$

Proof. Recall that

$$
F(\xi, \lambda \pm i \eta)=W(\xi, \lambda \pm i \eta) \exp \left\{\frac{1}{2 \pi i} \int_{a}^{b} \log \left|\frac{A(\mu)-\xi-\varepsilon k(\mu)}{A(\mu)-\xi+\varepsilon k(\mu)}\right| \frac{d \mu}{\mu-\lambda \pm i \eta}\right\}
$$

Thus we may employ Jensen's generalization of the inequality between arithmetic and geometric means [16] to conclude that

$$
|F(\xi, \lambda+i \eta)| \leqq|W(\xi, \lambda+i \eta)| \frac{\eta}{\pi} \int_{-\infty}^{\infty} \sqrt{ }\left(\left|\frac{A(\mu)-\xi-\varepsilon k(\mu)}{A(\mu)-\xi+\varepsilon k(\mu)}\right|\right) \frac{d \mu}{(\mu-\lambda)^{2}+\eta^{2}}
$$

where the square root in the integrand is defined to be identically equal to 
one when $\mu \notin(a, b)$. But since $\eta$ and $\delta$ are positive, we can use Fubini's theorem to write

$$
\begin{aligned}
\frac{\eta}{\pi} \int_{\tau_{j}}\left(\frac{g(\xi)}{\lambda+i \delta-\lambda_{j}(\xi)} \frac{T(\xi, \lambda+i \eta)-S(\xi, \lambda+i \eta)}{\sqrt{ }(S(\xi, \lambda+i \eta) T(\xi, \lambda+i \eta))}\right. & \left.\cdot \int_{-\infty}^{\infty} \sqrt{ }\left(\left|\frac{A(\mu)-\xi-\varepsilon k(\mu)}{A(\mu)-\xi+\varepsilon k(\mu)}\right|\right) \frac{d \mu}{(\mu-\lambda)^{2}+\eta^{2}}\right) d \xi \\
= & \frac{\eta}{\pi} \int_{-\infty}^{\infty} \frac{d \mu}{(\mu-\lambda)^{2}+\eta^{2}} \int_{\tau_{j}} \frac{g(\xi)}{\lambda+i \delta-\lambda_{j}(\xi)} \frac{T(\xi, \lambda+i \eta)-S(\xi, \lambda+i \eta)}{\sqrt{S(\xi, \lambda+i \eta) T(\xi, \lambda+i \eta)}} \\
& \quad \sqrt{ }\left(\left|\frac{A(\mu)-\xi-\varepsilon k(\xi)}{A(\mu)-\xi+\varepsilon k(\xi)}\right|\right) d \xi \equiv \frac{\eta}{\pi} \int_{-\infty}^{\infty} \frac{d \mu}{(\mu-\lambda)^{2}+\eta^{2}} G(\lambda, \mu, \eta, \delta) .
\end{aligned}
$$

Now we will estimate $G(\lambda, \mu, \eta, \delta)$ for $\lambda, \mu$ in the interior of $[a, b]$.

(B)

$$
\begin{array}{r}
|G(\lambda, \mu, \eta, \delta)| \leqq \max _{v \in \tau_{j}}|g(v)| \int_{\tau_{j} \cap D(g)}\left|\frac{T(\xi, \lambda)-S(\xi, \lambda)}{\lambda+i \delta-\lambda_{j}(\xi)}\right| \sqrt{ }\left(\left|\frac{A(\mu)-\xi-\varepsilon k(\mu)}{A(\mu) M \xi+\varepsilon k(\mu)}\right|\right) \\
+\max _{v \in \tau_{j}}|g(v)| \int_{\tau_{j} \cap D(g)}\left|\frac{T(\xi, \lambda+i \eta)-S(\xi, \lambda+i \eta)-T(\xi, \lambda)+S(\xi, \lambda)}{\lambda+i \delta-\lambda_{j}(\xi)}\right| \\
\left.\cdot \sqrt{ }|| \frac{A(\mu)-\xi-\varepsilon k(\mu)}{A(\mu)-\xi+\varepsilon k(\mu)} \mid\right) \cdot \frac{d \xi}{\sqrt{ }|S(\xi, \lambda+i \eta) T(\xi, \lambda+i \eta)|}
\end{array}
$$

where $D(g)$ denotes the support of $g(\xi)$.

When $\lambda$ is interior to one of the intervals of subdivision of $[a, b]$, say $\left[\alpha_{\kappa}, \alpha_{\kappa+1}\right]$ there will be a corresponding point set $\left\{\xi_{\lambda}^{\alpha}\right\} \subset \tau_{j}$, consisting of a finite number of points, for which one of the functions $S_{\kappa}(\xi)-\lambda$ and $T_{\kappa}(\xi)-\lambda$ vanishes. Since the functions $S_{r}(\xi)$ and $T_{r}(\xi)$ take their values in $\left[\alpha_{r}, \alpha_{r+1}\right]$ we see that $S_{r}(\xi)-\lambda$ and $T_{r}(\xi)-\lambda$ are bounded below independently of $\xi$ for $\lambda \in\left[\alpha_{k}, \alpha_{\kappa+1}\right]$ as long as $r \neq \kappa$ and $r \neq \kappa \pm 1$. For these adjacent intervals we must consider what happens when $\lambda$ is an endpoint, say $\alpha_{\kappa+1}$. There will now be, in general, a whole interval of $\xi$ values on which one of $S_{\kappa}(\xi)$ or $T_{\kappa+1}(\xi)$ will constantly be equal to $\lambda$. Not more than one of these functions, however, can effectively behave in this way. For if $S_{\kappa}(\xi)=\alpha_{\kappa+1}$ and $T_{\kappa+1}(\xi)=\alpha_{\kappa+1}$, then cancellation would occur in $W(\xi, \lambda)$.

Now, when $\lambda$ varies in the interior of $\left(\alpha_{k}, \alpha_{\kappa+1}\right)$ the set of $\xi$ 's for which, say, $S_{\kappa}(\xi)-\lambda=0$ intersected with $D(g)$ is such that $\left|S_{\kappa}^{\prime}(\xi)\right|$ has a positive lower bound, $m$.

Let us note that $S_{\kappa}(\xi)-T_{\kappa}(\xi)$ has a positive lower bound on the same set and $\left|W(\xi, \lambda) /\left(\lambda+i \delta-\lambda_{j}(\xi)\right)\right|$ remains bounded as $\delta \rightarrow 0$ so long as $\xi \in \tau_{j}$. 
We will achieve our estimates by breaking the $\xi$ interval of integration into a sum of integrals of the form

$$
\int_{\alpha}^{\xi_{\lambda}^{\kappa}-\eta / m}+\int_{\xi_{\lambda}^{\kappa}-\eta / m}^{\xi_{\lambda}^{\kappa}}+\int_{\xi_{\lambda}^{\kappa}}^{\xi_{\lambda}^{\kappa}+\eta / m}
$$

where $\eta$ has been taken so small that if $\xi_{\dot{\lambda}}^{\kappa}$ is a zero of $S_{x}(\xi)-\lambda$ and $\hat{\xi}_{\lambda}^{\kappa}$ is either another zero of $S_{\kappa}(\xi)-\lambda$ or a zero of $T_{\kappa}(\xi)-\lambda$ then $\xi_{i}^{\kappa}+\eta / m<\hat{\xi}_{\lambda}^{\kappa}-\eta / m$. (There is an $\bar{\eta}$ independent of $\lambda$ such that all $\eta \leqq \bar{\eta}$ will accomplish this.) We will not treat all the possible cases here but will merely indicate what happens. Suppose we only consider the last integral explicitly, with the others being treated analogously.

By the mean value theorem we can set $S_{\kappa}(\xi)-\lambda=\left(\xi-\xi_{\lambda}^{\kappa}\right) S_{\kappa}^{\prime}(\xi)$ for some $\xi$ intermediate between $\xi$ and $\xi_{\lambda}^{\boldsymbol{x}}$. Then

$$
\frac{1}{\sqrt{ }\left|\lambda+i \eta-S_{\kappa}(\xi)\right|} \leqq \frac{1}{\sqrt{ }\left(\eta-\left(\xi-\xi_{\lambda}^{\kappa}\right) m\right)} \text { for } \xi_{\lambda}^{\kappa}<\xi<\xi_{\lambda}^{\kappa}+\eta / m
$$

and we can assert that there is a constant, $C$, such that

$$
\begin{aligned}
& \left.\int_{\xi_{\lambda}^{\kappa}}^{\xi_{\lambda}^{\kappa}+\eta / m}|g(\xi)|\left|\frac{\omega(\xi, \lambda)}{\lambda+i \delta-\lambda_{j}(\xi)}\right|\right) /\left|\frac{A(\mu)+\varepsilon k(\mu)-\xi}{A(\mu)-\varepsilon k(\mu)-\xi}\right| d \xi \\
& \leqq \frac{C}{\sqrt{\left(\left|\alpha_{\kappa+1}-\lambda-i \eta\right|\left|\alpha_{\kappa}-\lambda-i \eta\right|\right)}} \int_{\xi_{\lambda}^{\kappa}}^{\xi_{\lambda}^{\kappa}+\eta / m} \frac{d \xi}{\sqrt{\left(\left(\eta-\left(\xi-\xi_{\lambda}^{\kappa}\right) m\right)|A(\mu)-\varepsilon k(\mu)-\xi|\right)}} \\
& \equiv \frac{C}{\sqrt{\left(\left|\alpha_{\kappa+1}-\lambda-i \eta\right|\left|\alpha_{\kappa}-\lambda-i \eta\right|\right)}} J_{k}(\lambda, \mu, \eta) .
\end{aligned}
$$

There are now several cases that may arise depending upon the location of $\mu$. If $\mu$ is such that $A(\mu)-\varepsilon k(\mu)-\xi_{\lambda}^{\kappa}-\eta / m>0$, then

$$
J_{\kappa}(\lambda, \mu, \eta)=\frac{2}{\sqrt{m}} \log \left|\frac{\sqrt{ }\left(A(\mu)-\varepsilon k(\mu)-\xi_{\lambda}^{\kappa}-\eta / m\right)}{\sqrt{(\eta / m)-\sqrt{ }\left(A(\mu)-\varepsilon k(\mu)-\xi_{\lambda}^{\kappa}\right)}}\right|
$$

but if $A(\mu)-\varepsilon k(\mu) \leqq \xi_{\lambda}^{\kappa}$, then

$$
J_{\kappa}(\lambda, \mu, \eta)=\frac{\pi}{\sqrt{ } m}-\frac{2}{\sqrt{ } m} \tan ^{-1} \sqrt{\left(\frac{m\left(\xi_{\lambda}^{\kappa}-A(\mu)+\varepsilon k(\mu)\right)}{\eta}\right)}
$$

and if, finally, $\xi(\mu) \equiv A(\mu)-\varepsilon k(\mu)$ is interior to the integration interval, then

$$
J_{\kappa}(\lambda, \mu, \eta)=\frac{\pi}{\sqrt{ } m}+\frac{2}{\sqrt{ } m} \log \left|\frac{\left.\sqrt{ }\left(\eta-A(\mu)-\varepsilon k(\mu)-\xi_{\lambda}^{\kappa}\right) m\right)}{\sqrt{\eta}-\sqrt{ } m\left(\left(A(\mu)-\varepsilon k(\mu)-\xi^{*}\right)\right.}\right| .
$$

Thus, in all of these cases, $J_{\kappa}(\lambda, \mu, \eta)$ is bounded, say by $M$, for all values of $\lambda, \mu, \eta)$ with $\lambda \in\left(\alpha_{k}, \alpha_{k+1}\right), \mu \in(a, b)$, and $\eta \leqq \bar{\eta}$. 
We may therefore conclude that there exists a constant $C_{\delta}$ such that

$$
\begin{array}{r}
\frac{\eta}{\pi} \int_{-\infty}^{\infty} \frac{d \mu}{(\mu-\lambda)^{2}+\eta^{2}}|N(\lambda, \mu, \eta, \delta)| \leqq \frac{C_{\delta}(1+\eta) M}{\sqrt{\left(\left|\alpha_{k}-\lambda-i \eta\right|\left|\alpha_{k+1}-\lambda-i \eta\right|\right)},} \\
\lambda \in(a, b),
\end{array}
$$

where $N(\lambda, \mu, \eta, \delta)$ is the maximum taken over $\kappa$ of the contributions to $G(\lambda, \mu, \eta, \delta)$ from the integration interval just considered, and the inequality follows because

$$
\frac{\eta}{\pi} \int_{-\infty}^{\infty} \frac{|f(\mu)|}{(\mu-\lambda)^{2}+\eta^{2}} d \mu \leqq \max |f(\lambda)|
$$

for any continuous $f(\lambda)$.

Using analogous results for the other ranges of integration, and adding all of them together, we will finally be able to assert that there exists a constant $G_{\delta}$ such that

$$
\int_{a}^{b}\left|M_{\eta, \delta}^{j}(g)\right|^{r} d \lambda<G_{\delta}
$$

for some $r$ in $1<r<2$ and fixed $\delta>0$.

Then we may use a result on uniform absolute continuity [17] to conclude that

$$
\int_{a}^{b} \lim _{\eta \downarrow 0}\left|M_{\eta, \delta}^{j}(g)\right|^{r} d \lambda<\infty,
$$

and

$$
\lim _{\eta^{\prime} \downarrow 0} \int_{a}^{b}\left|M_{\eta^{\prime}, \delta}^{j}(g)-\lim _{\eta \downarrow 0} M_{\eta, \delta}^{j}(g)\right| d \lambda=0 .
$$

Now let us note that the dependence of $G_{\delta}$ on $\delta$ comes from the second term in (B). We have used the Lipschitz continuity of $T(\xi, z)-S(\xi, z)$ in $z$ and taken a $\delta$-dependent lower bound for $\left[\lambda+i \delta-\lambda_{j}(\xi)\right]^{-1}$. If, for a fixed $\delta$, we go to the $\eta$ limit, this second term will give a vanishing contribution to the estimate of $\lim _{\eta \downarrow 0}\left|M_{\eta, \delta}^{j}(g)\right|$ and we can find now an absolute constant $G$ such that

$$
\int_{a \eta \eta 0}^{b} \lim _{\eta \downarrow}\left|M_{\eta, \delta}^{j}(g)\right|^{r} d \lambda \leqq G .
$$

But we can then conclude that

and

$$
\int_{a}^{b}\left|M^{j}(g)\right|^{r} d \lambda \leqq G
$$

$$
\lim _{\delta \downarrow 0} \lim _{\eta \downarrow 0} \int_{a}^{b}\left|M_{\eta, \delta}^{j}(g)-M^{j}(g)\right| d \lambda=0 .
$$




\section{The representation theorem.}

THeOREM. Let $k_{1}(\lambda)$ and $k_{2}(\lambda) \in C^{1}$ and be positive on $[a, b]$; and let $k(\lambda)=k_{1}(\lambda) k_{2}(\lambda)$, and let the hypothesis of Lemma 1 be satisfied $\left(^{8}\right)$.

Then if we define the transformation $\mathscr{S}: L_{2}(a, b) \rightarrow \mathscr{H}^{*}$ generated by the formulae

$$
\mathscr{S}_{f}(\lambda)=\left\{g_{0}(\xi), \cdots, g_{m(\xi)-1}(\xi)\right\}=g(\xi)
$$

where $\mathscr{S}^{(j)} f(\lambda)=g_{j}(\xi)$,

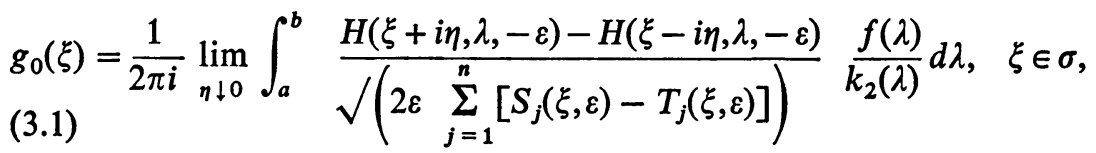

$g_{k}(\xi)=\frac{1}{2 \pi i} \lim _{\delta \downarrow 0} \lim _{\eta \downarrow 0} \int_{a}^{b} \frac{H(\xi+i \eta, \lambda,-\varepsilon)-H(\xi-i \eta, \lambda,-\varepsilon)}{\lambda-\lambda_{k}(\xi)+i \delta}$

$$
\sqrt{ }\left(\frac{\mu_{k}(\xi)}{2 \varepsilon}\right) \frac{f(\lambda)}{k_{2}(\lambda)} d \lambda, \xi \in \tau_{k}
$$

and for the transformation $\mathscr{T}: \mathscr{H}^{*} \rightarrow L_{2}(a, b)$ generated by the formula

$$
\begin{aligned}
& f(\lambda)=-\frac{1}{2 \pi i} \lim _{\delta^{\prime} \downarrow 0} \lim _{\eta^{\prime} \downarrow 0} \sum_{k=1}^{n-1} \int_{\tau_{k}} \frac{F\left(\xi, \lambda+i \eta^{\prime}, \varepsilon\right)-F\left(\xi, \lambda-i \eta^{\prime}, \varepsilon\right)}{\lambda-\lambda_{k}(\xi)+i \delta^{\prime}} \\
& \cdot \sqrt{ }\left(\frac{\mu_{k}(\xi)}{2 \varepsilon} \frac{g_{k}(\xi)}{k_{1}(\lambda)}\right) d \xi \\
&-\frac{1}{2 \pi i} \lim _{\eta^{\prime} \downarrow 0} \int_{\sigma} \frac{F\left(\xi, \lambda+i \eta^{\prime}, \varepsilon\right)-F\left(\xi, \lambda-i \eta^{\prime}, \varepsilon\right)}{\sqrt{ } 2 \varepsilon \sum_{j=1}^{n}\left(\left[S_{j}(\xi, \varepsilon)-T_{j}(\xi, \varepsilon)\right]\right)} \frac{g_{0}(\xi)}{k_{1}(\lambda)} d \xi
\end{aligned}
$$

we will have: $\mathscr{S}$ is a map of $L_{2}(a, b)$ onto $\mathscr{H}^{*}$ such that

and

$$
\mathscr{S} \mathscr{T}=I \text { and } \mathscr{T} \mathscr{S}=I \text {, }
$$

with

$$
\|\boldsymbol{g}(\cdot)\|_{\mathscr{H}^{*}}=\int_{a}^{b} \frac{k_{2}(\lambda)}{k_{1}(\lambda)}|f(\lambda)|^{2} d \lambda
$$

where

$$
\mathscr{S}\left(L_{\varepsilon} f(\cdot)\right)=\left\{\xi g_{0}(\xi), \cdots, \xi g_{m(\xi)-1}(\xi)\right\} \equiv \xi g(\xi)
$$

$$
L_{\varepsilon} x(\lambda)=A(\lambda) x(\lambda)+\frac{\varepsilon}{\pi i} \mathrm{P} \int_{a}^{b} \frac{k_{1}(\lambda) k_{2}(\mu)}{\mu-\lambda} x(\mu) d \mu .
$$

(8) Somewhat more is proved: namely, the theorem holds for complex-valued coefficients $k(\lambda, \mu)$ in case the $k(\lambda, \mu)=k(\lambda) k(\mu)$. 
Proof. We will show first that $\mathscr{S} \mathscr{T}=I$. Suppose that the components of $g(\xi)$ are each in $C^{\infty}(\sigma)$ and vanish in a neighborhood of the points $R^{ \pm}\left(\alpha_{k}\right), R^{ \pm}\left(\alpha_{k+1}\right)$; then we will show that we will have, for $\xi \in \tau_{k}, k=1, \cdots, n-1\left({ }^{9}\right)$

$$
\begin{aligned}
& g_{k}(\xi)=\frac{1}{2 \pi i} \lim _{\delta \downarrow 0} \lim _{\eta \downarrow 0} \int_{a}^{b} \frac{H(\xi+i \eta, \lambda,-\varepsilon)-H(\xi-i \eta, \lambda,-\varepsilon)}{\lambda-\lambda_{k}(\xi)+i \delta} \sqrt{\left(\frac{\mu_{k}(\xi)}{2 \varepsilon}\right) \frac{d \lambda}{k_{2}(\lambda)}} \\
& \cdot\left\{-\frac{1}{2 \pi i} \lim _{\delta^{\prime} \downarrow 0} \lim _{\eta^{\prime} \downarrow 0} \sum_{r=1}^{n-1} \int_{\tau_{r}} \frac{F\left(\gamma, \lambda+i \eta^{\prime}, \varepsilon\right)-F\left(\gamma, \lambda-i \eta^{\prime}, \varepsilon\right)}{\lambda-\lambda_{r}(\gamma)+i \delta^{\prime}} \sqrt{\left(\frac{\mu_{r}(\gamma)}{2 \varepsilon}\right) \frac{g_{r}(\gamma)}{k_{1}(\lambda)} d \gamma}\right.
\end{aligned}
$$

$$
\left.-\frac{1}{2 \pi i} \lim _{\eta^{\prime} \downarrow 0} \int_{\sigma} \frac{F\left(\gamma, \lambda+i \eta^{\prime}, \varepsilon\right)-F\left(\gamma, \lambda-i \eta^{\prime}, \varepsilon\right)}{\sqrt{ }\left(2 \varepsilon \sum_{j=1}^{n}\left[S_{j}(\gamma, \varepsilon)-T_{j}(\gamma, \varepsilon)\right]\right)} \frac{g_{0}(\gamma)}{k_{1}(\lambda)} d \gamma\right\}
$$

In order to prove this assertion, let us consider the quantity, for $l=\xi+i \eta$; $\eta>0, \delta>0$

$$
I_{j}^{m}(l) \equiv \frac{1}{2 \pi i} \lim _{\delta \downarrow 0} \lim _{\eta \downarrow 0} \int_{a}^{b} \frac{H(l, \lambda,-\varepsilon)}{\lambda-\lambda_{j}(\xi)+i \delta} \sqrt{ } /\left(\frac{\mu_{j}(\xi)}{2 \varepsilon} \frac{d \lambda}{k_{2}(\lambda)}\right)
$$

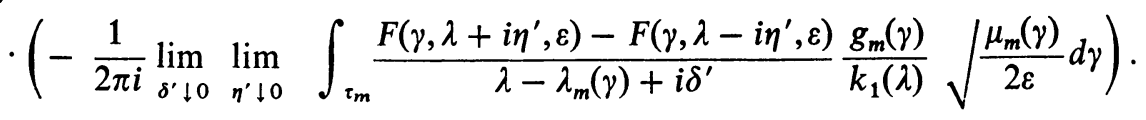

Now by Lemma 4 we know that the following expression

$$
\int_{a}^{b} d \lambda\left|\int_{\tau_{m}} \frac{F\left(\gamma, \lambda \pm i \eta^{\prime}, \varepsilon\right)}{\lambda-\lambda_{m}(\gamma)+i \delta^{\prime}} g_{m}(\gamma) d \gamma\right|^{r} \leqq G, \text { for an } r>1,
$$

has boundedness properties which enable us to conclude [17] that the $\delta^{\prime}$ and $\eta^{\prime}$ limits can be interchanged with the $\lambda$-integration. If we do this, and use Fubini's theorem we will have

$$
\begin{aligned}
I_{j}^{m}(l)=\frac{1}{4 \pi^{2}} \lim _{\delta \downarrow 0} \lim _{\eta \downarrow 0} \lim _{\delta^{\prime} \downarrow 0} \lim _{\eta^{\prime} \downarrow 0} \int_{\tau_{m}} \sqrt{ }\left(\mu_{m}(\gamma) \mu_{j}(\xi)\right) g_{m}(\gamma) d \gamma \\
\cdot \int_{a}^{b} \frac{H(l, \lambda,-\varepsilon)\left[F\left(\gamma, \lambda+i \eta^{\prime}, \varepsilon\right)-F\left(\gamma, \lambda-i \eta^{\prime}, \varepsilon\right)\right]}{\left[\lambda-\lambda_{j}(\xi)+i \delta\right]\left[\lambda-\lambda_{m}(\gamma)+i \delta^{\prime}\right]} \frac{d \lambda}{2 \varepsilon k(\lambda)} .
\end{aligned}
$$

But let us note [18] that if $L(\lambda)$ and $J(\gamma)$ are continuous, then for $r>1$ we can use a well-known inequality to assert that

(9) We will not explicitly treat the other cross terms; they can be evaluated in an analogous way. 


$$
\begin{aligned}
\left(\int_{\tau}|J(\gamma)|^{r} d \gamma \quad\left|\int_{a}^{b} L(\lambda) \frac{F\left(\gamma, \lambda \pm i \eta^{\prime}, \varepsilon\right)}{\lambda+i \delta^{\prime}-\lambda_{m}(\gamma)} d \lambda\right|^{r}\right)^{1 / r} \\
\quad \leqq \int_{a}^{b}\left(\int_{\tau_{m}}\left|L(\lambda) \frac{F\left(\gamma, \lambda \pm i \eta^{\prime}, \varepsilon\right)}{\lambda+i \delta^{\prime}-\lambda_{m}(\gamma)}\right|^{r}|J(\gamma)|^{r} d \gamma\right)^{1 / r} d \lambda
\end{aligned}
$$

and thus we can again use uniform absolute continuity to exchange the $\delta^{\prime}$ and $\eta^{\prime}$ limits in (3.5) with the $\gamma$ integration.

If we do this, and then invoke Lemma 2 and formula (1.8) we can conclude that

$$
\begin{aligned}
& I_{j}^{m}(l)=\lim _{\delta \downarrow 0} \lim _{\eta \downarrow 0} \frac{1}{4 \pi^{2}} \int_{\tau_{m}} \sqrt{ }\left(\mu_{m}(\gamma) \mu_{j}(\xi)\right) g_{m}(\gamma) d \gamma \\
& \cdot \lim _{\delta^{\prime} \rightarrow 0} \lim _{\eta^{\prime} \rightarrow 0} \int_{a}^{b} \frac{H(l, \lambda,-\varepsilon)\left[H\left(\gamma+i \eta^{\prime}, \lambda, \varepsilon\right)-H\left(\gamma-i \eta^{\prime}, \lambda, \varepsilon\right)\right]}{\left[\lambda-\lambda_{j}(\xi)+i \delta\right]\left[\lambda-\lambda_{m}(\gamma)+i \delta^{\prime}\right]} \frac{d \lambda}{2 \varepsilon k(\lambda)} .
\end{aligned}
$$

Thus let us examine

$$
\frac{1}{2 \pi i} \int_{a}^{b} \frac{H(l, \lambda,-\varepsilon) H\left(\gamma+i \eta^{\prime}, \lambda, \varepsilon\right)}{\left[\lambda-\lambda_{j}(\xi)+i \delta\right]\left[\lambda-\lambda_{m}(\gamma)+i \delta^{\prime}\right]} \frac{d \lambda}{2 \varepsilon k(\lambda)}
$$

which, by virtue of (2.16), can be written

$$
=\frac{1}{2 \pi i} \frac{1}{l-\gamma-i \eta^{\prime}} \oint_{C} \frac{E(l, z,-\varepsilon) E\left(\gamma+i \eta^{\prime}, z, \varepsilon\right)}{\left[z-\lambda_{j}(\xi)+i \delta\right]\left[z-\lambda_{m}(\gamma)+i \delta^{\prime}\right]} d z
$$

where $C$ is the contour of Figure 1.

But by the residue theorem this last expression is equal to

$$
\begin{aligned}
\frac{1}{l-\gamma-i \eta^{\prime}} & \left\{\frac{1}{\lambda_{m}(\gamma)+i \delta-i \delta^{\prime}-\lambda_{j}(\xi)} E\left(l, \lambda_{m}(\gamma)-i \delta^{\prime},-\varepsilon\right) E\left(\gamma+i \eta^{\prime}, \lambda_{m}(\gamma)-i \delta^{\prime}, \varepsilon\right)\right. \\
& \left.+\frac{1}{\lambda_{j}(\xi)+i \delta-i \delta^{\prime}-\lambda_{m}(\gamma)} E\left(l, \lambda_{j}(\xi)-i \delta,-\varepsilon\right) E\left(\gamma+i \eta^{\prime}, \lambda_{j}(\xi)-i \delta, \varepsilon\right)\right\} .
\end{aligned}
$$

Now subtract the same expression with $-\eta^{\prime}$ and go to the $\eta^{\prime}$ limit to get

$$
\begin{aligned}
\frac{1}{l-\gamma}\left\{\left[\frac{E\left(l, \lambda_{m}(\gamma)-i \delta^{\prime},-\varepsilon\right)}{\lambda_{m}(\gamma)+i \delta+i \delta^{\prime}-\lambda_{j}(\xi)}\left[E^{+}\left(\gamma, \lambda_{m}(\gamma)-i \delta^{\prime}, \varepsilon\right)-E^{-}\left(\gamma, \lambda_{m}(\gamma)-i \delta^{\prime}, \varepsilon\right)\right]\right.\right. \\
\left.\left.\quad+\frac{E\left(l, \lambda_{j}(\xi)-i \delta,-\varepsilon\right)}{\lambda_{j}(\xi)+i \delta+i \delta^{\prime}-\lambda_{m}(\gamma)}\left[E^{+}\left(\gamma, \lambda_{j}(\xi)-i \delta, \varepsilon\right)-E^{-}\left(\gamma, \lambda_{j}(\xi)-i \delta, \varepsilon\right)\right]\right]\right\}
\end{aligned}
$$

and if we take the $\delta^{\prime}$ limit, we obtain

$$
\frac{1}{l-\gamma}\left\{\frac{E_{-}\left(l, \lambda_{j}(\xi)-i \delta,-\varepsilon\right)}{\lambda_{j}(\xi)+i \delta-\lambda_{m}(\gamma)}\left[E^{+}\left(\gamma, \lambda_{j}(\xi)-i \delta\right)-E^{-}\left(\gamma, \lambda_{j}(\xi)-i \delta\right)\right]\right\} .
$$


Let us note for future use that this iterated limit is equal to the limit obtained by setting $\eta^{\prime}=\delta^{\prime} \rightarrow 0$.

We have now obtained

$$
\begin{aligned}
I_{j}^{m}(l)=\lim _{\delta \downarrow 0} \lim _{\eta \downarrow 0} \frac{1}{4 \pi^{2}} \int_{\tau_{m}} \frac{\sqrt{ }\left(\mu_{m}(\gamma) \mu_{j}(\xi)\right)}{l-\gamma} g_{m}(\gamma) d \gamma \\
\cdot\left\{\frac{E^{-}\left(l, \lambda_{j}(\xi)-i \delta,-\varepsilon\right)}{\lambda_{j}(\xi)-i \delta-\lambda_{m}(\gamma)} F\left(\gamma, \lambda_{j}(\xi)-i \delta\right)\right\} .
\end{aligned}
$$

Let us denote the quantity whose limit is being taken by $J_{j}^{m}(l)$.

We wish to evaluate

$$
J_{j}^{m}(\xi+i \eta)-J_{j}^{m}(\xi-i \eta) \text { in the limit } \eta \rightarrow 0 .
$$

Now

$$
\begin{aligned}
\lim _{\delta \downarrow 0} \lim _{\eta \downarrow 0} J_{j}^{m}(\xi+i \eta)=E_{+}^{-}\left(\xi, \lambda_{j}(\xi),-\varepsilon\right) \lim _{\delta \downarrow 0} \lim _{\eta \downarrow 0} \frac{1}{4 \pi^{2}} \int_{\tau_{m}} \frac{\sqrt{ }\left(\mu_{m}(\gamma) \mu_{j}(\xi)\right)}{\xi+i \eta-\gamma} \\
\cdot g_{m}(\gamma) \frac{F\left(\gamma, \lambda_{j}(\xi)-i \delta\right)}{\lambda_{j}(\xi)-i \delta-\lambda_{m}(\gamma)} d \gamma
\end{aligned}
$$

$+\lim _{\delta \downarrow 0} \lim _{\eta \downarrow 0}\left[E^{-}\left(\xi+i \eta, \lambda_{j}(\xi)-i \delta,-\varepsilon\right)-E_{+}^{-}\left(\xi, \lambda_{j}(\xi)-i \delta,-\varepsilon\right)\right] \frac{1}{4 \pi^{2}} \int_{\tau_{m}} \frac{\sqrt{ }\left(\mu_{m}(\gamma) \mu_{j}(\xi)\right)}{\xi+i \eta-\gamma}$

$$
g_{m}(\gamma) \frac{F\left(\gamma, \lambda_{j}(\xi)-i \delta\right)}{\lambda_{j}(\xi)-i \delta-\lambda_{m}(\gamma)} d \gamma
$$

But for each fixed positive $\delta$, the continuity properties of the integrand imply that the $\eta$-limit of the integral exists and is finite for each $\xi$. Hence we can conclude that

$$
\begin{aligned}
& \lim _{\delta \downarrow 0} \lim _{\eta \downarrow 0} J_{j}^{m}(\xi+i \eta)=E_{+}^{-}\left(\xi, \lambda_{j}(\xi),-\varepsilon\right) \lim _{\delta \downarrow 0} \lim _{\gamma \downarrow 0} \frac{1}{4 \pi^{2}} \int_{\tau_{m}} \frac{\sqrt{ }\left(\mu_{m}(\gamma) \mu_{j}(\xi)\right)}{\xi+i \eta-\gamma} g_{m}(\xi) \\
& \cdot \frac{F\left(\gamma, \lambda_{j}(\xi)-i \delta\right)}{\lambda_{j}(\xi)-i \delta-\lambda_{m}(\gamma)} d \gamma .
\end{aligned}
$$

Finally, if we use the Plemelj-Privalov theorem in conjunction with the fact that $F\left(\xi, \lambda_{j}(\xi),-\varepsilon\right)=0$, we can get

$$
\begin{array}{r}
\lim \lim _{\delta \downarrow 0}\left(J_{j \downarrow 0}^{m}(\xi+i \eta)-J_{j}^{m}(\xi-i \eta)\right)=\lim _{\delta \downarrow 0} \lim _{\gamma \rightarrow \xi}\left[\frac{F\left(\gamma, \lambda_{j}(\xi)-i \delta\right)}{\lambda_{j}(\xi)+i \delta-\lambda_{m}(\gamma)}\right] g_{m}(\xi) \\
\cdot \sqrt{ }\left(\mu_{m}(\xi) \mu_{j}(\xi)\right)\left[E_{+}^{-}\left(\xi, \lambda_{j}(\xi),-\varepsilon\right)+E_{-}^{-}\left(\xi, \lambda_{j}(\xi),-\varepsilon\right)\right]
\end{array}
$$

and if we now use relations (1.6) and (2.9) together with 


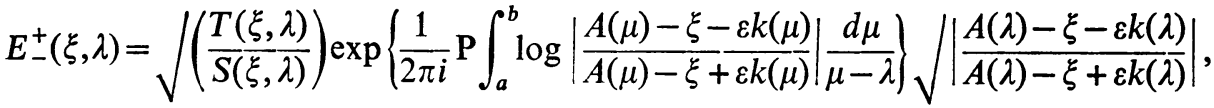

$$
\begin{aligned}
& E_{-}^{-}(\xi, \lambda)=\int\left(\frac{S(\xi, \lambda)}{T(\xi, \lambda)}\right) \exp \left\{\frac{1}{2 \pi i} \mathrm{P} \int_{a}^{b} \log \left|\frac{A(\mu)-\xi-\varepsilon k(\mu)}{A(\mu)-\xi+\varepsilon k(\mu)}\right| \frac{d \mu}{\mu-\lambda}\right\} \sqrt{\left|\frac{A(\lambda)-\xi-\varepsilon k(\lambda)}{A(\lambda)-\xi+\varepsilon k(\lambda)}\right|}
\end{aligned}
$$

and do a little bookkeeping, we can conclude that

$$
\begin{aligned}
\lim _{\delta \downarrow 0} \lim _{\gamma \rightarrow \xi}\left[\frac{F\left(\gamma, \lambda_{j}(\xi)-i \delta\right)}{\lambda_{j}(\xi)+i \delta-\lambda_{m}(\gamma)}\right]\left(E _ { + } ^ { - } \left(\xi, \lambda_{j}(\xi),\right.\right. & \left.-\varepsilon)+E_{-}^{-}\left(\xi, \lambda_{j}(\xi),-\varepsilon\right)\right) \\
& =\left\{\begin{array}{cc}
\frac{1}{\mu_{m}(\xi)} & \text { if } j=m, \\
0 & \text { if } j \neq m,
\end{array}\right.
\end{aligned}
$$

and thus

$$
\lim _{\delta \downarrow 0} \lim _{\eta \downarrow 0}\left(J_{j}^{m}(\xi+i \eta)-J_{j}^{m}(\xi-i \eta)\right)=\left\{\begin{array}{cc}
g_{m}(\xi), & j=m, \\
0, & j \neq m .
\end{array}\right.
$$

While this calculation shows that $\mathscr{S} \mathscr{T}=I$ when the domain of $\mathscr{T}$ is suffciently restricted, it does not show that the image of even such a restricted domain in $\mathscr{H}^{*}$ lies in $L_{2}(a, b)$.

We will now establish that $\mathscr{T}$ generates an isometry mapping $\mathscr{H}^{*}$ onto $L_{2}(a, b)$.

In the following for ease of presentation, and without loss of generality, we will take $L_{\varepsilon}$ self-adjoint so that $k_{1}(\lambda)=k_{2}(\lambda)=\sqrt{ } k(\lambda)$.

Now consider the transformation $\mathscr{S}$ defined by relation (3.1) and let us evaluate the image of a dense set of the form $\{\sqrt{ }(2 \varepsilon k(\lambda)) /(\lambda-z)\}$ (where $z$ ranges to infinity).

Then we can use the residue theorem to assert that

$$
\frac{1}{2 \pi i} \int_{a}^{b} \frac{H(\xi+i \eta, \mu,-\varepsilon)}{\mu+i \delta-\lambda_{j}(\xi)} \frac{d \mu}{\mu-z}=\frac{E(\xi+i \eta, z,-\varepsilon)}{z+i \delta-\lambda_{j}(\xi)}+\frac{E\left(\xi+i \eta, \lambda_{j}(\xi)-i \delta,-\varepsilon\right)}{\lambda_{j}(\xi)-i \delta-z}
$$

and thus

$$
\mathscr{S}\left\{\frac{\sqrt{ }(2 \varepsilon k(\lambda))}{\lambda-z}\right\}=g_{z}=\left\{\frac{F(\xi, \bar{z})}{B(\xi) / 2 \varepsilon}, \frac{\bar{F}(\xi, \bar{z})}{z-\lambda_{1}(\xi)} \sqrt{ } \mu_{1}(\xi), \cdots, \frac{F(\xi, \bar{z})}{z-\lambda_{m(\xi)}(\xi)} \sqrt{ } \mu_{m(\xi)}(\xi)\right\} .
$$

But $U_{\text {extended }}^{-1}\left(g_{z}\right)=\sqrt{ }(2 \varepsilon k(\lambda)) /(\lambda-z)$, and $U_{\text {extended }}^{-1}$ is, as previously remarked, an isometry on its domain.

Thus $\mathscr{S}$ may be uniquely extended to an isometry on all of $L_{2}(a, b)$. Let us explicitly note here that exactly the same extension would be obtained if in the definition of $\mathscr{S}$ we had set $\eta=\delta$ or replaced $\delta$ by $-\delta$. Furthermore let us explicitly point out that $\mathscr{S}$ can also be uniquely extended to all of $L_{1}(a, b)$ since $(a, b)$ is a finite interval.

Now consider the expression 


$$
\int_{\tau_{m}} \overline{g_{m}(\xi)} \mathscr{S}^{(m)} \mathscr{T}\left(g_{0}, \cdots, g_{n(\gamma)}\right) d \xi=\int_{\tau_{m}}\left|g_{m}(\xi)\right|^{2} d \xi
$$

where the $g$ 's are chosen as before. We have seen that we can take $\eta^{\prime}=\delta^{\prime}$ in the definition of $\mathscr{T}$ without disturbing this equality, and we now consider $\mathscr{T}$ to be taken in this way. Similarly, we can take $\delta=-\eta$ in the definition of $\mathscr{S}^{(m)}$.

Then

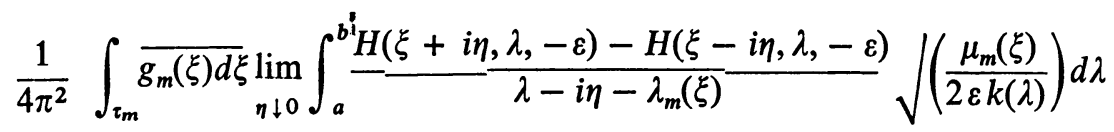

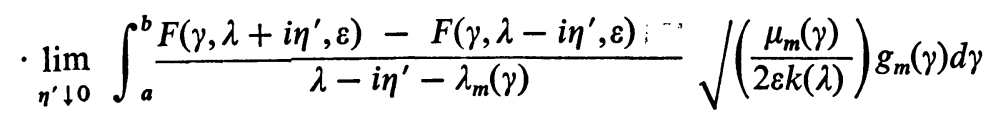

$$
\begin{aligned}
& =\int_{\tau_{m}}\left|g_{m}(\xi)\right|^{2} d \xi \text {. }
\end{aligned}
$$

But we have already justified writing the left-hand side of this equality in the form

$$
\begin{array}{r}
\frac{1}{4 \pi^{2}} \int_{\tau_{m}} \overline{g_{m}(\xi)} d \xi \lim _{\eta \downarrow 0 \eta^{\prime} \downarrow 0} \int_{\tau_{m}} \sqrt{ }\left(\mu_{m}(\gamma) \mu_{j}(\xi)\right) g_{m}(\gamma) d \gamma \cdot \int_{a}^{b} \frac{d \lambda}{2 \varepsilon k(\lambda)} \\
\frac{H(\xi+i \eta, \lambda,-\varepsilon)-H(\xi-i \eta, \lambda,-\varepsilon)}{\lambda-\lambda_{m}(\xi)-i \eta} \cdot \frac{H\left(\gamma+i \eta^{\prime}, \lambda, \varepsilon\right)-H\left(\gamma-i \eta^{\prime}, \lambda, \varepsilon\right)}{\lambda-\lambda_{m}(\gamma)+i \eta^{\prime}}
\end{array}
$$

and we can utilize the explicit calculation which we have made for the $\lambda$ integral to justify a final exchange of limits with the $\xi$ integration. Then if we apply Fubini's theorem we will have

$$
\begin{aligned}
& \int_{\tau_{m}}\left|g_{m}(\xi)\right|^{2} d \xi=\lim _{\eta \downarrow 0} \lim _{\eta^{\prime} \downarrow 0} \frac{1}{4 \pi^{2}} \int_{a}^{b} d \lambda \int_{\tau_{m}} \frac{H(\xi+i \eta, \lambda,-\varepsilon)-H(\xi-i \eta, \lambda,-\varepsilon)}{\lambda-\lambda_{m}(\xi)-i \eta} \\
& \cdot \sqrt{\left(\frac{\mu_{m}(\xi)}{2 \varepsilon k(\lambda)}\right) \overline{g_{m}(\xi)} d \xi} \\
& \cdot \int_{\tau_{m}} \frac{H(\gamma+i \eta, \lambda, \varepsilon)-H(\gamma-i \eta, \lambda, \varepsilon)}{\lambda-\lambda_{m}(\gamma)+i \eta^{\prime}} \sqrt{\left(\frac{\mu_{m}(\gamma)}{2 \varepsilon k(\lambda)}\right) g_{m}(\gamma) d \gamma .}
\end{aligned}
$$

But by (1.8) this last expression is nothing but

$$
\begin{aligned}
& \lim \lim _{\eta \downarrow 0} \frac{1}{4 \eta^{\prime} \downarrow 0} \int_{a}^{b} d \lambda \overline{\int_{\tau_{m}} \frac{H(\xi+i \eta, \lambda, \varepsilon)-H(\xi-i \eta, \lambda, \varepsilon)}{\lambda-\lambda_{m}(\xi)+i \eta} \sqrt{\left(\frac{\mu_{m}(\xi)}{2 \varepsilon k(\lambda)}\right) g_{m}(\xi) d \xi}} \\
& \cdot \int_{\tau_{m}} \frac{H\left(\gamma+i \eta^{\prime}, \lambda, \varepsilon\right)-H\left(\gamma-i \eta^{\prime}, \lambda, \varepsilon\right)}{\lambda-\lambda_{m}(\gamma)+i \eta^{\prime}} \sqrt{\left(\frac{\mu_{m}(\gamma)}{2 \varepsilon k(\lambda)}\right) g_{m}(\gamma) d \gamma}
\end{aligned}
$$

and this is, with obvious notation, 


$$
\lim _{\eta \downarrow 0} \lim _{\eta^{\prime} \downarrow 0} \int_{a}^{b} \overline{\mathscr{T}_{\eta}\left(0, \cdots, g_{m}, 0, \cdots, 0\right)} \mathscr{T}_{\eta^{\prime}}\left(0, \cdots, g_{m}, 0, \cdots, 0\right) d \lambda .
$$

However,

$$
\begin{aligned}
\lim _{\eta \downarrow 0} & \lim _{\eta^{\prime} \downarrow 0} \int_{a}^{b} d \lambda \overline{\mathscr{T}_{\eta}\left(g_{0}, g_{1}, \cdots, g_{n}\right)} \mathscr{T}_{\eta^{\prime}}\left(g_{0}, \cdots, g_{n}\right) \\
& =\lim _{\eta \downarrow 0} \lim _{\eta^{\prime} \downarrow 0} \sum_{m=1}^{n} \int_{a}^{b} d \lambda \overline{\mathscr{T}_{\eta}\left(0, \cdots, 0, g_{m}, 0, \cdots, 0\right)} \mathscr{T}_{\eta^{\prime}}\left(0, \cdots, 0, g_{m}, 0, \cdots, 0\right)
\end{aligned}
$$

because, as we have seen, the cross products vanish in the limit.

Hence we have deduced that

$$
\int_{\sigma} \sum_{0}^{m(\xi)-1}\left|g_{j}(\xi)\right|^{2} d \xi=\lim _{\eta \downarrow 0} \lim _{\eta^{\prime} \downarrow 0} \int_{a}^{b} \overline{\mathscr{T}_{\eta}\left(g_{0}, \cdots, g_{n}\right)} \mathscr{T}_{\eta^{\prime}}\left(g_{0}, \cdots, g_{n}\right) d \lambda .
$$

However, it is still not possible to assert that this proves that $\lim _{\eta \downarrow 0} \mathscr{T}_{\eta}\left(g_{0}, \cdots, g_{n}\right) \in L_{2}(a, b)$, because we cannot directly justify an exchange of both the limits in the above equality with the $\lambda$-integration.

Accordingly we will proceed by first showing that the iterated limit can be replaced by a double limit, and consequently we can proceed to zero with $\eta=\eta^{\prime}$. Then we will have a non-negative integrand and will be able to use Fatou's theorem to assert that

$$
\int_{a}^{b}\left|\mathscr{T}\left(g_{0}, \cdots, g_{n}\right)\right|^{2} d \lambda \leqq \int_{\sigma} \sum_{0}^{m(\xi)-1}\left|g_{j}(\xi)\right|^{2} d \xi
$$

In order to implement this plan, we will show that the original iterated limit could have been replaced by a double limit, i.e.,

$$
\begin{array}{r}
\lim _{\eta \downarrow 0} \lim _{\eta^{\prime} \downarrow 0} \int_{\tau_{m}} \overline{g_{m}(\xi)} \mathscr{S}_{\eta}^{(m)} \mathscr{T}_{\eta^{\prime}} g_{m} d \xi \\
=\lim _{\eta \downarrow 0} \int_{\tau_{m}} \overline{g_{m}(\xi)} \mathscr{S}_{\eta}^{(m)} \mathscr{T}_{\eta} g_{m} d \xi .
\end{array}
$$

Consider therefore

$$
\lim _{\eta^{\prime} \downarrow 0} \mathscr{S}_{\eta}^{(m)}\left(\mathscr{T}_{\eta^{\prime}}-\mathscr{T}\right)\left(g_{0}, \cdots, g_{n}\right) .
$$

Since we have shown in Lemma 4 that

$$
\lim _{\eta^{\prime} \downarrow 0} \int_{a}^{b}\left|\left(\mathscr{T}_{\eta^{\prime}}-\mathscr{T}\right)\left(g_{0}, \cdots, g_{n}\right)\right| d \lambda=0
$$

for the $g$ 's under consideration, we may invoke the Banach-Steinhaus uniform boundedness theorem [19] on $L_{1}(a, b)$ to assert that 


$$
\lim _{\eta^{\prime} \downarrow 0} \mathscr{S}_{\eta_{\eta}}^{(m)}\left(\mathscr{T}_{\eta^{\prime}}-\mathscr{T}\right)\left(g_{0}, \cdots, g_{n}\right)=0
$$

uniformly in $\eta$. But this can happen only if $\lim _{\eta^{\prime} \downarrow 0} \mathscr{S}_{\eta}^{(m)} \mathscr{T}_{\eta^{\prime}}\left(g_{0}, \cdots, g_{n}\right)$ is assumed uniformly in $\eta$.

Thus we have now satisfied the conditions for the Moore-Osgood theorem to apply, and can accordingly assert the equality of the double and iterated limits; and, finally, can use Fatou's theorem.

We have therefore proved that $\mathscr{T}\left(g_{0}, \cdots, g_{n}\right) \in L_{2}(a, b)$, and it remains to demonstrate that $\mathscr{T}$ is isometric on this set of $g$ 's.

For this purpose recall that

$$
\int_{\sigma}^{m(\xi)-1} \sum_{0}\left|g_{j}(\xi)\right|^{2} d \xi=\lim _{\eta} \lim _{\eta^{\prime}} \int_{a}^{b^{\prime \prime}} \overline{\mathscr{T}_{\eta}\left(g_{0}, \cdots, g_{n}\right)} \mathscr{T}_{\eta^{\prime}}\left(g_{0}, \cdots, g_{n}\right) d \lambda .
$$

For a fixed $\eta, \mathscr{T}_{\eta}\left(g_{0}, \cdots, g_{n}\right)$ defines a continuous linear functional on $L_{2}(a, b)$ by the Frechet-Riesz theorem. Hence

$$
\int_{0}^{\mathbf{I}_{m(\xi)}} \sum_{0}\left|g_{j}(\xi)\right|^{2} d \xi=\lim _{\eta} \int_{a}^{b} \overline{\mathscr{T}_{\eta}\left(g_{0}, \cdots, g_{n}\right)} \mathscr{T}\left(g_{0}, \cdots, g_{n}\right) d \lambda
$$

but, since $\mathscr{T}\left(g_{0}, \cdots, g_{n}\right) \in L_{2}(a, b)$, this function also defines a continuous linear functional, and we can finally assert that

$$
\int_{0}^{m(\xi)-1} \sum_{j=0}^{m}\left|g_{j}(\xi)\right|^{2} d \xi=\int_{a}^{b}\left|\mathscr{T}\left(g_{0}, \cdots, g_{n}\right)\right|^{2} d \lambda
$$

Since the set of $g$ 's for which this is true is dense in $\mathscr{H}^{*}$ there exists a unique isometric extension for $\mathscr{T}$ defined on all of $\mathscr{H}^{*}$.

We must now show that $\mathscr{T} \mathscr{S}=I$ on $L_{2}(a, b)$. In order to accomplish this let us recall that (in the notation of Lemma 2)

$$
\mathscr{S}\left(\mathscr{T}-U_{\text {extended }}^{-1}\right) D\left(U_{\text {extended }}^{-1}\right)=0
$$

since $U_{\text {extended }}^{-1}\left(g_{z}\right)=\sqrt{ }(2 \varepsilon k(\lambda)) /(\lambda-z)$ and $\mathscr{S}(\sqrt{ }(2 \varepsilon k(\lambda)) /(\lambda-z))=g_{z}$. But, since $\mathscr{S}$ has an inverse we can conclude from this that $\mathscr{T}=U_{\text {extended }}^{-1}$ on the domain of $U_{\text {extended. But we have seen that }}^{-1}$

$$
U_{\text {extended }}^{-1} \mathscr{S}\left(\frac{\sqrt{ }(2 \varepsilon k(\lambda))}{\lambda-z}\right)=\frac{\sqrt{ }(2 \varepsilon k(\lambda))}{\lambda-z},
$$

and the range of $\mathscr{S}$ is just $D\left(U_{\text {extended }}^{-1}\right)$. Hence $\mathscr{T} \mathscr{S}=I$ on all of $L_{2}(a, b)$.

Now $\mathscr{T}$ is an isometric mapping from $\mathscr{H}^{*}$ onto $L_{2}(a, b)$. The images of the $g_{z}$ are dense in $L_{2}(a, b)$ and $\mathscr{S}$ is the inverse of $\mathscr{T}$ hence the range of $\mathscr{S}$ must be all of $\mathscr{H}^{*}$.

Now let us show that 
if

$$
\mathscr{S}\left(L_{\varepsilon} f(\cdot)\right)=\left\{\xi g_{0}(\xi), \cdots, \xi g_{m(\xi)}(\xi)\right\}
$$

$$
\mathscr{S} f(\cdot)=\left\{g_{0}(\xi), \cdots, g_{m(\xi)}(\xi)\right\} \text {. }
$$

To do this, let $f(\mu)$ again be chosen from the dense set $\{\sqrt{ }(2 \varepsilon k(\mu)) /(\mu-z)\}$, for $z$ real and outside $(a, b)$. Then we wish to calculate (for $\xi \in \tau_{\kappa}$ )

$$
\begin{aligned}
& \frac{1}{2 \pi i} \lim _{\delta \downarrow 0} \lim _{\eta \downarrow 0} \int_{a}^{b} \frac{H(\xi+i \eta, \lambda,-\varepsilon)-H(\xi-i \eta, \lambda,-\varepsilon)}{\lambda-\lambda(\xi)+i \delta} \sqrt{\left(\frac{\mu_{\kappa}(\xi)}{2 \varepsilon k(\lambda)}\right) \cdot L_{\varepsilon}\left[\frac{\sqrt{ }(2 \varepsilon k(\mu))}{\mu-z}\right] d \lambda} \\
& \quad=\frac{1}{2 \pi i} \lim _{\delta \downarrow 0} \lim _{\eta \downarrow 0}\left(L_{\varepsilon}\left(\frac{\sqrt{ }(2 \varepsilon k(\mu))}{\mu-z}\right) \frac{H(\xi+i \eta, \lambda, \varepsilon)-H(\xi-i \eta, \lambda, \varepsilon)}{\lambda-\lambda_{\kappa}(\xi)+i \delta} \sqrt{\left.\left(\frac{\mu_{\kappa}(\xi)}{2 \varepsilon k(\lambda)}\right)\right)}\right.
\end{aligned}
$$

where $(f, g)=\int_{a}^{b} f(\lambda) \overline{g(\lambda)} d \lambda$ and where we have replaced $\delta$ by $-\delta$ in $\mathscr{S}$ as is permitted.

But, since $L_{\varepsilon}$ is self-adjoint on $L_{2}(a, b)$, this expression is equal to

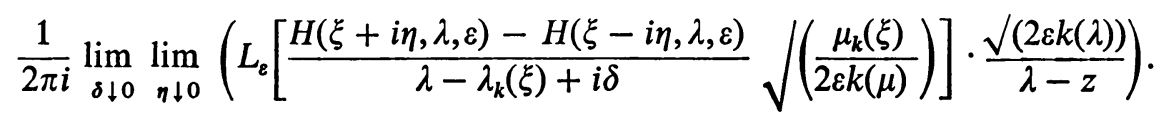

But if we recall that

$$
\frac{1}{2 \pi i} \int_{a}^{b} \frac{H(\xi+i \eta, \mu, \varepsilon)}{\mu+i \delta-\lambda_{j}(\xi)} \frac{d \mu}{\mu-z}=\frac{E(\xi+i \eta, z, \varepsilon)}{z+i \delta-\lambda_{j}(\xi)}+\frac{E\left(\xi+i \eta, \lambda_{j}(\xi)-i \delta, \varepsilon\right)}{\lambda_{j}(\xi)-i \delta-z}
$$

and use the slight modification of (1.18) relevant to the case where

$$
L_{\varepsilon} x(\lambda)=A(\lambda) x(\lambda)+\frac{\varepsilon}{\pi i} \int_{a}^{b} \frac{\sqrt{ }(k(\lambda) k(\mu))}{\mu-\lambda} x(\mu) d \mu
$$

together with

$\lim _{\delta \downarrow 0} \lim _{\eta \downarrow 0} 2\left(E\left(\xi+i \eta, \lambda_{j}(\xi)-i \delta\right)-E\left(\xi-i \eta, \lambda_{j}(\xi)-i \delta\right)\right) \int_{a}^{b} \frac{d \mu}{\lambda_{j}(\xi)-i \delta-\mu} \frac{1}{\mu-z}=0$

then the desired conclusion follows by a direct evaluation for functions of our special class. Now let $f(\lambda)$ be any function of class $L_{2}(a, b)$. Then we can approximate $f(\lambda)$ by sums, $\sum c_{p} /\left(\lambda-z_{p}\right)=f_{n}(\lambda)$, so that $\lim _{n \rightarrow \infty} f_{n}=f$ in the norm of $L_{2}(a, b)$. Let us call $\mathscr{S} f_{n}=\left(g_{0}^{(n)}(\xi), \cdots, g_{m(\xi)}^{(n)}(\xi)\right) \equiv g^{(n)}$. Because $\mathscr{S}$ is an isometry $\mathscr{S} f_{n} \rightarrow \mathscr{S} f \equiv\left(g_{0}(\xi), \cdots, g_{m(\xi)}(\xi)\right)$. But because our $\xi$ interval is bounded, the functions $\xi g_{j}^{(n)}(\xi)$ converge to $\xi g_{j}(\xi)$ in the $L_{2}(\sigma)$ norm, and hence $\xi g^{(n)} \equiv\left(\xi g_{0}^{(n)}(\xi), \cdots, \xi g_{m(\xi)}(\xi)\right)$ converges to $\xi g(\xi) \equiv\left(\xi g_{0}(\xi), \cdots, \xi g_{m(\xi)}(\xi)\right)$ in the $\mathscr{H}^{*}$ norm. Now since $f_{n}(\lambda)$ is a linear combination of functions in our special class $\mathscr{S}\left(L_{\varepsilon} f_{n}\right)=\left(g_{0}^{(n)}(\xi), \cdots, \xi g_{m(\xi)}^{(n)}(\xi)\right)$. But $L_{\varepsilon}$ is continuous in the norm of $L_{2}(a, b)$. Hence $\lim _{n \rightarrow \infty} L_{\varepsilon} f_{n}=L_{\varepsilon} f$. Thus if we let $h(\lambda)=\mathscr{T}\left\{\xi g_{0}(\xi), \cdots, \xi g_{m(\xi)}(\xi)\right\}$ $=\mathscr{T}(\xi g)$, we may write 


$$
\begin{aligned}
\left\|h(\lambda)-L_{\varepsilon} f(\lambda)\right\|_{L_{2}(a, b)} & \leqq\left\|h(\lambda)-L_{\varepsilon} f_{n}(\lambda)\right\|_{L_{2}(a, b)}+\left\|L_{\varepsilon} f_{n}(\lambda)-L_{\varepsilon} f(\lambda)\right\|_{L_{2}(a, b)} \\
& =\left\|\xi g(\xi)-\xi g^{(n)}(\xi)\right\|_{\mathscr{P}^{*}}+\left\|L_{\varepsilon} f_{n}(\lambda)-L_{\varepsilon} f(\lambda)\right\|_{L_{2}(a, b)}
\end{aligned}
$$

and if we let $n \rightarrow \infty$, we get $h(\lambda)=L_{\varepsilon} f(\lambda)$, i.e., $\mathscr{T}(\xi g(\xi))=L_{\varepsilon} f(\lambda)$ or $\xi \boldsymbol{g}(\xi)=\mathscr{S}\left(L_{\varepsilon} f(\lambda)\right)$.

The theorem is now completely proven except in the non-self-adjoint case when

$$
L_{\varepsilon} x(\lambda)=A(\lambda) x(\lambda)+\frac{\varepsilon}{\pi i} \mathrm{P} \int_{a}^{b} \frac{k_{1}(\lambda) k_{2}(\mu)}{\mu-\lambda} \dot{x}(\mu) d \mu .
$$

However, although $L_{\varepsilon}$ is not self-adjoint it is symmetrizable [20] and can be rendered self-adjoint with respect to a new scalar product derived from the $L_{2}(a, b)$ scalar product by multiplication by a simple combination of the functions $\sqrt{ } k_{1}(\lambda)$ and $\sqrt{ } k_{2}(\lambda)$. The remaining assertions of the theorem then furnish the spectral representation of this new $L_{\varepsilon}$, considered as self-adjoint, on such a new Hilbert space. These results follow in an entirely trivial way from the preceding ones and their proof will be omitted here.

It is a pleasure to acknowledge a conversation with $L$. de Branges and the advice and encouragement of J. W. Calkin. Finally, the author wishes to express his gratitude to $K$. O. Friedrichs for valuable suggestions and special encouragement to pursue this work.

Added in proof. A connection has now been obtained [21] between the analytic machinery developed here and the multiplicity theory of generalized eigen-functions for arbitrary self-adjoint operators with absolutely continuous spectra lutely continuous spectra defined over $\mathscr{L}^{2}\left(E^{n}\right)$.

\section{REFERENCES}

1. D. Hilbert, Grundzüge eine allgemeinen Theorie der linearen Integralgleichungen, Teubner, Leipzig, 1912; reprinted Chelsea, New York, 1952.

2. H. Poincaré, Leçons de mécanique céleste, Vol. 3, Gauthier-Villars, Paris, 1910.

3. T. Carleman, Sur la résolution de certaines équations intégrales, Ark. Mat. Astronom. Fys. 16 (1922).

4. N. I. Muskhelishvili, Singular integral equations, Noordhoff, Groningen, 1953.

5. I. N. Vekua (See references and author's index in reference 4).

6. H. Widom, Singular integral equations in $L_{p}$, Trans. Amer. Math. Soc. 97 (1960), 131-160.

7. J. Schwartz, Some results on the spectra and spectral resolutions of a class of singular integral operators, Comm. Pure Appl. Math. 15 (1962), 75-90.

8. W. Koppelman and J. Pincus, Perturbation of continuous spectra and singular integral operators, Institute of Mathematical Sciences Rep. No. 242, New York Univ., New York.

9. - Spectral representations for finite Hilbert transformations, Math. Z. 71 (1959), 399-407.

10. W. Koppelman, On the spectral theory of singular integral operators, Trans. Amer. Math. Soc. 97 (1960), 35-63.

11. J. Pincus, Thesis, New York Univ., New York, 1959. 
12. K. O. Friedrichs, Perturbation of continuous spectra, Comm. Pure Appl. Math. 1 (1948), $361-406$.

13. - Uber die Spektralzerlegung eines integral Operators, Math. Ann. 115 (1938), 249-272.

14. H. G. Tillmann, Vector valued distributions and the spectral theorem for selfadjoint operators in Hilbert space, Bull. Amer. Math. Soc. 69 (1963), 63-67.

15. N. I. Ahiezer and I. M. Glassmann, Theorie der linearen operatoren im Hilbert-Raum, p. 38, Akademie-Verlag, Berlin, 1954.

16. G. H. Hardy, J. E. Littlewood and G. Pólya, Inequalities, p. 137, Cambridge Univ. Press, Cambridge, 1952.

17. J. Littlewood, Theory of functions, p. 40, Oxford Univ. Press, Oxford, 1944.

18. G. H. Hardy, J. E. Littlewood and G. Pólya, Inequalities, p. 148, Cambridge Univ. Press, Cambridge, 1952.

19. N. Dunford and J. Schwartz, Linear operators. I, p. 54, Interscience, New York, 1958.

20. P. Lax, Symmetrizable linear transformations, Comm. Pure Appl. Math. 7 (1954), 633-647.

21. J. Pincus, On the construction of generalized eigenfunction expansions, Brookhaven National Laboratory Rep. No. 7963, Upton, L.I., N.Y.

BROOKHAVEN NATIONAL LABORATORY,

UPTON, L. I., NEW YORK 